\title{
Symmetric Meso-Mechanical Model for Failure Analysis of Heterogeneous Materials
}

\author{
Robert Crouch and Caglar Oskay* \\ Department of Civil and Environmental Engineering \\ Vanderbilt University \\ Nashville, TN, 37235
}

\begin{abstract}
This manuscript provides a novel reduced-order multiscale modeling methodology for failure analysis of heterogeneous materials. The proposed methodology is based on the computational homogenization method for bridging multiple spatial scales and the eigendeformation-based model reduction method to incorporate failure in the microconstituents and interfaces. This computationally-efficient modeling methodology leads to symmetric reduced-order algebraic systems for evaluation of the microscale boundary value problem. The order and coarse graining for the reduced-order system are systematically identified by a novel model development strategy. Verification studies reveal that the proposed methodology efficiently and accurately models the failure response. The proposed approach eliminates the spurious residual stress effect observed in reduced-order models, which pollutes the post-failure stress field at the macroscale.
\end{abstract}

Keywords: reduced-order; homogenization; multiscale; failure; composites

\section{Introduction}

Mathematical homogenization theory provides a rigorous mathematical framework for modeling the response of heterogeneous materials. The mathematical theory was formalized in the seminal works of Babuska [1], Bensoussan [2], Sanchez-Palencia [3] and Suquet [4], among others. Since the development of the computational framework for the mathematical homogenization theory by Guedes and Kikuchi [5], numerous models based on the computational homogenization method (CHM) have been proposed to predict the elastic and inelastic response of heterogeneous materials including material failure.

The distinct feature of the computational homogenization method in modeling the response of heterogeneous materials is in the evaluation of the constitutive response at a material point of a macroscopic (homogenized) medium. In CHM, the constitutive response of the equivalent

${ }^{*}$ Corresponding Author: VU Station B\#351831, 2301 Vanderbilt Place, Nashville, TN $37235 . \quad$ Email: caglar.oskay@vanderbilt.edu 
homogeneous medium is evaluated by solving a microscale boundary value problem defined on a representative volume element (RVE) of the heterogeneous microstructure. This approach decouples the effect of the microstructural topology from the material behavior of the microconstituents, as well as the conditions along the microconstituent interfaces. CHM simplifies the constitutive modeling process since the response of the microconstituents tend to be simpler to model, compared to phenomenological modeling of the combined microstructure-material behavior effects. In the case of modeling the failure of heterogeneous materials, a number of outstanding computational issues remain, including selection of the boundary conditions for the RVE problem in the presence of defects [6,7], evolution of the RVE domain upon defect formation, size scale effects [8], and spurious mesh dependency [9], among others.

One additional major challenge associated with the computational homogenization method is the computational cost associated with solving nonlinear RVE problems to evaluate the constitutive response of the macroscopic problem. This problem is alleviated by one or a combination of two approaches. The first is the brute-force parallelization of the multiscale problem, in which, the RVE problem evaluations are distributed to a large number of compute nodes and evaluated in parallel [10]. The second approach is reduced-order evaluation of the RVE problem. Fast Fourier transform [11], proper orthogonal decomposition [12], spectral method [13], boundary element method [14], network approximation method [15], and transformation field analysis (TFA) [16, 17], and other TFA-based computational methods [18, 19, 20] have been effective in evaluating the inelastic response at the RVE level in a computationally efficient manner. In a recent study, eigendeformation-based homogenization method (EHM) was proposed [21] to efficiently evaluate the RVE level response using a meso-mechanical model. This method is derived based on a generalization of the transformation field analysis. By this approach, it is possible to account for the interfacial debonding effects, in addition to nonlinear and failure processes within the constituent materials of the heterogeneous microstructure.

This manuscript provides a model reduction methodology for efficient evaluation of the microscale boundary value problems of the computational homogenization method. The proposed approach addresses three of the main shortcomings of the TFA-based model reduction methods with the the following novel contributions:

1. A new methodology for the determination of the order of the reduced model is presented: The accuracy and efficiency of the reduced models clearly depend on their order and ability to represent the failure modes within the microstructure. A reduced-order model development strategy is devised to identify the model order and the associated coarse graining at the microscale for accurate and efficient representation of the failure modes.

2. The proposed reduced order model leads to a symmetric formulation: In the presence of interfacial debonding, previous eigendeformation-based homogenization formulations lack symmetry, which increases computational cost.

3. The proposed formulation eliminates the spurious residual stress effect upon failure due to the coarse representation of the inelastic fields. Some of the transformation field analysis based reduced order models (e.g., [18, 21]) lead to spurious residual stress fields upon failure in the microscale. The spurious residual stress fields pollute the macroscale problem by affecting the local stress redistributions.

The proposed reduced order methodology is implemented to model the failure response of brittle composite systems, in which the failure is characterized by matrix microcracking, delamination and debonding. 


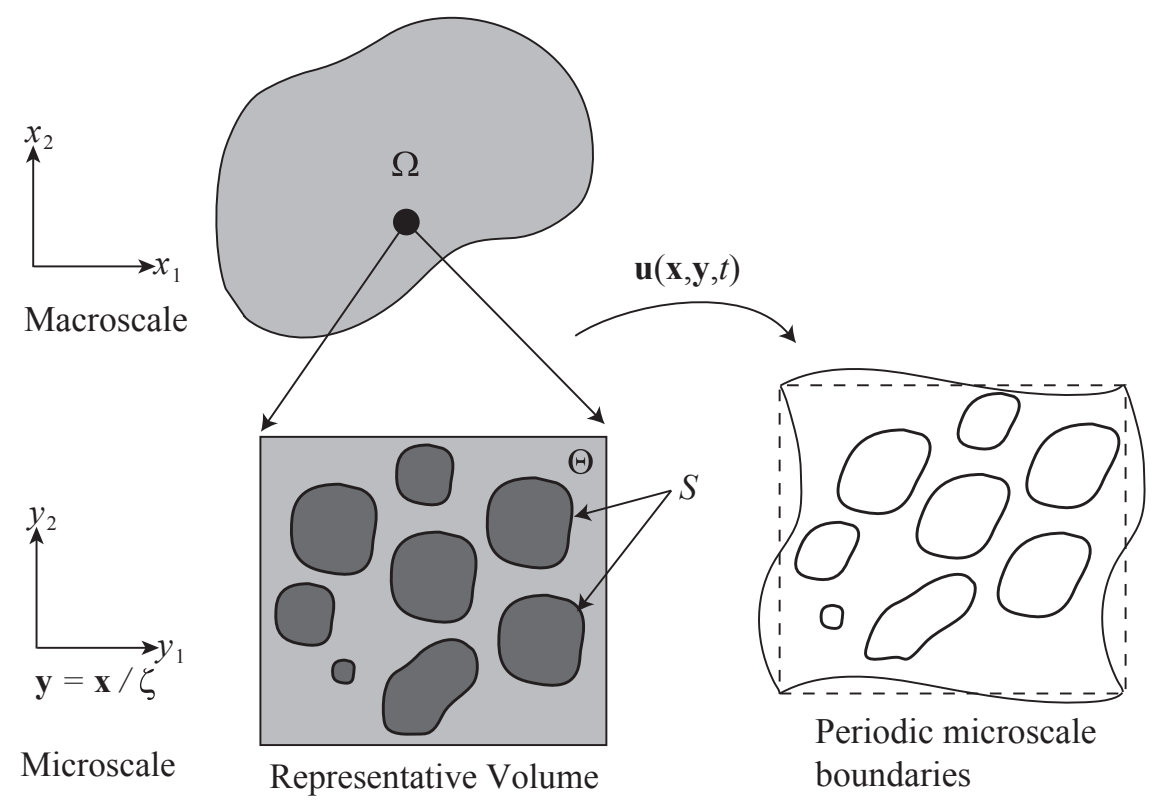

Figure 1: Macro- and microscopic scales.

The remainder of this manuscript is organized as follows: The statement of the multiscale problem and the associated macroscopic and microscopic boundary value problems are presented in Section 2. In Section 3, formulation of the symmetric reduced order model for the microscale problem is provided. The computational algorithms employed to evaluate the nonlinear reduced order model are discussed in Section 4. Section 5 provides small scale and large scale numerical verification examples conducted on a fiber reinforced matrix composite. Conclusions and discussion of future research is discussed in Section 6.

\section{Problem Setting}

In this section, we present a summary of the microscopic and macroscopic boundary value problems associated with the two-scale asymptotic homogenization method for failure response of a heterogeneous body. The details of two-scale asymptotic homogenization in the presence of inelastic effects are reported in the literature (see e.g., Refs. [22]).

The problem setting and the multiscale heterogeneous body is illustrated in Fig. 1. The heterogeneous domain, denoted by $\Omega$, is parameterized by the macroscopic coordinate vector, $\mathbf{x} . \quad \Omega$ is composed of the repetition of a small representative volume element, $\Theta$, which is parameterized by the microscopic coordinate vector, $\mathbf{y}$. The size scale ratio, $\zeta$, between the characteristic lengths of the representative volume element, $\Theta$, and the macroscopic body, $\Omega$ is assumed to be very small, such that a first order asymptotic decomposition of the displacement field is sufficient to accurately capture the response of the material. The response fields are assumed to be periodic about the representative volume element. The periodicity condition states that the value of the response fields are the same at the opposing faces of a parallelepiped RVE domain.

The following notation is employed throughout the manuscript, unless otherwise noted: Subscript roman indices denote 1, 2, or 3. Einstein summation convention is adopted for repeated indices. Subscripts $x_{i}$ and $y_{i}$ following a comma denote differentiation with respect 
to the macroscopic and microscopic coordinate vectors, respectively. Differentiation within parentheses denotes symmetric differentiation with respect to the indices. Bold characters denote tensor notation. Macaulay brackets denote averaging over the RVE:

$$
\langle\cdot\rangle=\frac{1}{|\Theta|} \int_{\Theta}(\cdot) d \mathbf{y}
$$

where, $|\Theta|$ is the volume of the RVE.

The displacement field of the heterogeneous body is expressed using a two-scale asymptotic expansion:

$$
u_{i}(\mathbf{x}, \mathbf{y}, t)=\bar{u}_{i}(\mathbf{x}, t)+\zeta u_{i}^{1}(\mathbf{x}, \mathbf{y}, t)
$$

in which, $\overline{\mathbf{u}}$ is the macroscopic displacement field, and; $\mathbf{u}^{1}$ is the variation of the displacement field within the RVE.

\subsection{Microscale Problem}

In the presence of failure processes, $\mathbf{u}^{1}$ is described by the microscopic equilibrium equation defined over the RVE (i.e., $\mathbf{y} \in \Theta$ )

$$
\left\{L_{i j k l}(\mathbf{y})\left[\bar{\epsilon}_{k l}(\mathbf{x}, t)+u_{\left(k, y_{l}\right)}^{1}(\mathbf{x}, \mathbf{y}, t)-\mu_{k l}(\mathbf{x}, \mathbf{y}, t)\right]\right\}_{, y_{j}}=0
$$

in which, $\mathbf{L}$ is the fourth order tensor of elastic moduli, taken to be symmetric and strongly elliptic, $\overline{\boldsymbol{\epsilon}}=\nabla_{\mathbf{x}}^{s} \overline{\mathbf{u}}$ the macroscopic strain tensor; $\nabla_{\mathbf{x}}^{s}(\cdot) \equiv(\cdot)_{\left(i, x_{j}\right)}$ denotes the symmetric gradient operation with respect to macroscopic coordinates; and $\boldsymbol{\mu}$ damage induced inelastic strains. In this manuscript, the damage induced inelastic strains are modeled using a scalar continuous damage mechanics model:

$$
\mu_{i j}(\mathbf{x}, \mathbf{y}, t)=\left[1-\omega_{\mathrm{ph}}(\mathbf{x}, \mathbf{y}, t)\right] \epsilon_{i j}(\mathbf{x}, \mathbf{y}, t)
$$

in which, $\omega_{\mathrm{ph}} \in[0,1)$ is a history dependent variable, which represents damage within the microconstituents, and $\boldsymbol{\epsilon}$ is the strain tensor. Using the scaling relations provided by the asymptotic decompositions with multiple spatial scales:

$$
\epsilon_{i j}(\mathbf{x}, \mathbf{y}, t)=\bar{\epsilon}_{i j}(\mathbf{x}, t)+u_{\left(i, y_{j}\right)}^{1}(\mathbf{x}, \mathbf{y}, t)
$$

Along the microconstituent interfaces, debonding is considered based on traction-separation laws given as $(\mathbf{y} \in S)$

$$
\begin{array}{r}
t^{N}(\mathbf{x}, \mathbf{y}, t)-\left[1-\omega_{\text {int }}(\mathbf{x}, \mathbf{y}, t)\right] k^{N}(\mathbf{y}) \delta^{N}(\mathbf{x}, \mathbf{y}, t) \leq 0 ; \quad \delta^{N}(\mathbf{x}, \mathbf{y}, t) \geq 0 \\
\left\{t^{N}(\mathbf{x}, \mathbf{y}, t)-\left[1-\omega_{\text {int }}(\mathbf{x}, \mathbf{y}, t)\right] k^{N}(\mathbf{y}) \delta^{N}(\mathbf{x}, \mathbf{y}, t)\right\} \delta^{N}(\mathbf{x}, \mathbf{y}, t)=0 \\
\mathbf{t}^{T}(\mathbf{x}, \mathbf{y}, t)=\left[1-\omega_{\text {int }}(\mathbf{x}, \mathbf{y}, t)\right] k^{T}(\mathbf{y}) \boldsymbol{\delta}^{T}(\mathbf{x}, \mathbf{y}, t)
\end{array}
$$

in which, $\omega_{\text {int }} \in[0,1)$ is a history dependent variable, which represents damage along the interface; $t^{N}$ and $\delta^{N}$ are the components of the traction and displacement jump normal to the interface, respectively; $k^{N}(\mathbf{y})$ and $k^{T}(\mathbf{y})$ the initial interface stiffness in the normal and tangential directions, respectively, and; $\mathbf{t}^{T}, \boldsymbol{\delta}^{T}$ the tangential components of the traction and displacement jump along the interface, respectively. The traction and displacement jump components are expressed in terms of the local coordinate system formed by the normal and tangential directions at the interface point. 
The microscale problem, which is a nonlinear boundary value problem is solved to evaluate the microscale displacement field $\mathbf{u}^{1}$ by imposing periodic boundary conditions along the exterior boundaries of the RVE while restricting the rigid body motion. The microscale boundary value problem is quasi-static as indicated by the lack of inertial terms in the governing equations. The present formulation is limited to the cases for which the characteristic size of the RVE is small compared to the length of the deformation and stress waves.

\subsection{Macroscale Problem}

The macroscopic displacement field is described by the macroscopic momentum balance equation defined over $\Omega$ :

$$
\bar{\sigma}_{i j, x_{j}}(\mathbf{x}, t)+\bar{b}_{i}(\mathbf{x}, t)=\bar{\rho}(\mathbf{x}, t) \ddot{\bar{u}}_{i}(\mathbf{x}, t)
$$

in which, double dot over a field denotes twice differentiation in time; $\overline{\boldsymbol{\sigma}}$ denotes the macroscopic stress tensor, evaluated by volume averaging of the stresses over the domain of the RVE

$$
\bar{\sigma}_{i j}(\mathbf{x}, t)=\left\langle\sigma_{i j}\right\rangle
$$

The stress field is expressed as:

$$
\sigma_{i j}(\mathbf{x}, \mathbf{y}, t)=L_{i j k l}(\mathbf{y})\left[\bar{\epsilon}_{k l}(\mathbf{x}, t)+u_{\left(k, y_{l}\right)}^{1}(\mathbf{x}, \mathbf{y}, t)-\mu_{k l}(\mathbf{x}, \mathbf{y}, t)\right]
$$

$\overline{\mathbf{b}}$ and $\bar{\rho}$ denote the RVE-average body force/unit volume and the RVE-average density, respectively:

$$
\bar{b}_{i}(\mathbf{x}, t)=\left\langle b_{i}\right\rangle ; \quad \bar{\rho}=\langle\rho\rangle
$$

The boundary and initial conditions of the macroscale initial-boundary value problem are defined as

$$
\begin{aligned}
\bar{u}_{i}(\mathbf{x}, t) & =\hat{u}_{i}(\mathbf{x}) ; \quad \mathbf{x} \in \Omega ; \quad t=0 \\
\dot{\bar{u}}_{i}(\mathbf{x}, t) & =\hat{v}_{i}(\mathbf{x}) ; \quad \mathbf{x} \in \Omega ; \quad t=0 \\
\bar{u}_{i}(\mathbf{x}, t) & =\breve{u}_{i}(\mathbf{x}, t) ; \quad \mathbf{x} \in \Gamma_{u} ; \quad t \in\left[0, t_{\mathrm{o}}\right] \\
\bar{\sigma}_{i j}(\mathbf{x}, t) n_{j} & =\breve{t}_{i}(\mathbf{x}, t) ; \quad \mathbf{x} \in \Gamma_{t} ; \quad t \in\left[0, t_{\mathrm{o}}\right]
\end{aligned}
$$

in which, $\hat{\mathbf{u}}, \breve{\mathbf{u}}$ are prescribed initial and boundary displacements, respectively; $\hat{\mathbf{v}}$ prescribed initial velocity, and; $\breve{\mathbf{t}}$ prescribed boundary traction. The prescribed initial and boundary conditions are assumed to be constant with respect to the microscopic coordinate vector $\mathbf{y}$.

\section{Reduced Order Modeling of the Microscale Problem}

The macroscale problem defined in Section 2.2 is coupled with the microscale problem defined in Section 2.1 through the macroscopic constitutive relationship (Eqs. 10 and 11). The evaluation of the macroscopic stress at each macroscopic material point requires the solution of the microscopic RVE problem associated with that material point. When the finite element method is employed to evaluate the macroscale problem, a nonlinear microscale problem must be evaluated to update the stress at each integration point for each increment and iteration of every time step of the loading history. This is a tremendous computational burden. In this section, a novel reduced order model is derived to efficiently compute the microscopic response. 
To this extent, the microscale displacement field is decomposed into linear and damage induced components:

$$
u_{i}^{1}(\mathbf{x}, \mathbf{y}, t)=H_{i k l}(\mathbf{y}) \bar{\epsilon}_{k l}(\mathbf{x}, t)+\tilde{u}_{i}(\mathbf{x}, \mathbf{y}, t)
$$

in which, $\mathbf{H}$ is the third order elastic influence function obtained by substituting Eq. 17 into Eq. 3 and solving the microscale problem in the absence of all inelastic processes (i.e., $\left.\omega_{\mathrm{ph}}=\omega_{\text {int }}=0\right)$. $\quad \tilde{\mathbf{u}}$ is the displacement field induced by the damage processes within the microconstituents and the interface:

$$
\tilde{u}_{i}(\mathbf{x}, \mathbf{y}, t)=\int_{\Theta} h_{i k l}^{\mathrm{ph}}(\mathbf{y}, \hat{\mathbf{y}}) \mu_{k l}(\mathbf{x}, \hat{\mathbf{y}}, t) d \hat{\mathbf{y}}+\int_{S} h_{i m}^{\mathrm{int}}(\mathbf{y}, \hat{\mathbf{y}}) \delta_{m}(\mathbf{x}, \hat{\mathbf{y}}, t) d \hat{\mathbf{y}}
$$

in which $\mathbf{h}^{\mathrm{ph}}$ and $\mathbf{h}^{\text {int }}$ are the phase damage and interface damage induced influence functions. $\mathbf{h}^{\mathrm{ph}}$ and $\mathbf{h}^{\text {int }}$ are the particular solutions to the RVE problems obtained by substituting Eq. 17 into Eq. 3 and solving the microscale problem in the presence of phase damage (i.e., $\boldsymbol{\mu}$ ) and interface damage (i.e., $\boldsymbol{\delta}$ ), respectively. The governing equations and the discrete approximations of the elastic and damage induced influence functions are provided in Ref. [21] and will not be discussed herein. In this manuscript, we concentrate on the new model reduction methodology based on the microscopic displacement field decomposition provided in Eqs. 17 and 18.

Substituting Eq. 17 into Eq. 3, premultiplying the resulting equation with $\mathbf{h}^{\mathrm{ph}}$, and integrating over the domain of the RVE yields:

$$
\int_{\Theta} h_{i p q}^{\mathrm{ph}}(\mathbf{y}, \hat{\mathbf{y}})\left\{L_{i j m n}(\mathbf{y})\left[A_{m n k l}(\mathbf{y}) \bar{\epsilon}_{k l}(\mathbf{x}, t)+\tilde{\epsilon}_{m n}(\mathbf{x}, \mathbf{y}, t)-\mu_{m n}(\mathbf{x}, \mathbf{y}, t)\right]\right\}_{, y_{j}} d \mathbf{y}=0
$$

in which, $\tilde{\boldsymbol{\epsilon}}=\nabla_{\mathbf{y}}^{s} \tilde{\mathbf{u}} ; \mathbf{A}=\mathbf{I}+\mathbf{G}$ is the fourth order elastic strain concentration tensor; $\mathbf{I}$ the fourth order identity tensor, and; $\mathbf{G}=\nabla_{\mathbf{y}}^{s} \mathbf{H}$, the elastic polarization tensor. The use of Eq. 19 secures a symmetric formulation as subsequently derived. This is in contrast with the previous eigendeformation-based reduced order models, which are non-symmetric [21]. Integrating by parts, applying divergence theorem and employing the perodicity of the response fields over the domain of the RVE yields:

$$
\int_{\Theta} g_{i j p q}^{\mathrm{ph}}(\mathbf{y}, \hat{\mathbf{y}}) L_{i j m n}(\mathbf{y})\left[A_{m n k l}(\mathbf{y}) \bar{\epsilon}_{k l}(\mathbf{x}, t)+\tilde{\epsilon}_{m n}(\mathbf{x}, \mathbf{y}, t)-\mu_{m n}(\mathbf{x}, \mathbf{y}, t)\right] d \mathbf{y}=0
$$

where, $\mathbf{g}^{\mathrm{ph}}=\nabla_{\mathbf{y}}^{s} \mathbf{h}^{\mathrm{ph}}$ is the fourth order phase damage polarization tensor.

A second set of equilibrium equations are obtained by premultiplying the microscale equilibrium equation (Eq. 3) with $\mathbf{h}^{\text {int }}$, and following a similar procedure as described above:

$$
\int_{\Theta} g_{i j p}^{\mathrm{int}}(\mathbf{y}, \hat{\mathbf{y}}) L_{i j m n}(\mathbf{y})\left[A_{m n k l}(\mathbf{y}) \bar{\epsilon}_{k l}(\mathbf{x}, t)+\tilde{\epsilon}_{m n}(\mathbf{x}, \mathbf{y}, t)-\mu_{m n}(\mathbf{x}, \mathbf{y}, t)\right] d \mathbf{y}=-t_{p}(\mathbf{x}, \hat{\mathbf{y}}, t)
$$

in which, $\mathbf{g}^{\text {int }}=\nabla_{\mathbf{y}}^{s} \mathbf{h}^{\text {int }}$ is the third order interface damage polarization tensor. Substituting Eq. 4 into Eqs. 20 and 21 yields:

$$
\begin{gathered}
\int_{\Theta}\left[1-\omega_{\mathrm{ph}}(\mathbf{x}, \mathbf{y}, t)\right] g_{i j q r}^{\mathrm{ph}}(\mathbf{y}, \hat{\mathbf{y}}) L_{i j m n}(\mathbf{y}) \cdot \\
\cdot\left[A_{m n k l}(\mathbf{y}) \bar{\epsilon}_{k l}(\mathbf{x}, t)+\tilde{\epsilon}_{m n}(\mathbf{x}, \mathbf{y}, t)\right] d \mathbf{y}=0 \\
\int_{\Theta}\left[1-\omega_{\mathrm{ph}}(\mathbf{x}, \mathbf{y}, t)\right] g_{i j p}^{\mathrm{int}}(\mathbf{y}, \hat{\mathbf{y}}) L_{i j m n}(\mathbf{y}) \cdot \\
\cdot\left[A_{m n k l}(\mathbf{y}) \bar{\epsilon}_{k l}(\mathbf{x}, t)+\tilde{\epsilon}_{m n}(\mathbf{x}, \mathbf{y}, t)\right] d \mathbf{y}=-t_{p}(\mathbf{x}, \hat{\mathbf{y}}, t)
\end{gathered}
$$


We introduce the following discretizations for damage fields $\omega_{\mathrm{ph}}$ and $\omega_{\text {int }}$, and the damage induced fields, $\boldsymbol{\mu}$ and $\boldsymbol{\delta}$ using mesomechanical shape functions

$$
\begin{aligned}
& \left\{\omega_{\mathrm{ph}}, \mu_{i j}\right\}(\mathbf{x}, \mathbf{y}, t)=\sum_{\gamma=1}^{n} N_{\mathrm{ph}}^{(\gamma)}(\mathbf{y})\left\{\omega_{\mathrm{ph}}^{(\gamma)}, \mu_{i j}^{(\gamma)}\right\}(\mathbf{x}, t) \\
& \left\{\omega_{\text {int }}, \hat{\delta}_{i}\right\}(\mathbf{x}, \mathbf{y}, t)=\sum_{\beta=1}^{m} N_{\text {int }}^{(\beta)}(\mathbf{y})\left\{\omega_{\text {int }}^{(\beta)}, \hat{\delta}_{i}^{(\beta)}\right\}(\mathbf{x}, t)
\end{aligned}
$$

in which, $\gamma=1,2, \ldots, n$ and $\beta=1,2, \ldots, m ; n$ and $m$ denote the level of discretization within the phases and along the interface, respectively. $\hat{\boldsymbol{\delta}}$ denotes the displacement jump vector in the local coordinate system (i.e., $\hat{\delta}^{(\beta)}=\left[\delta^{N(\beta)} \boldsymbol{\delta}^{T(\beta)}\right]^{T}$ ). The phase, $N_{\mathrm{ph}}^{(\gamma)}$, and interface, $N_{\text {int }}^{(\beta)}$, shape functions have compact support within subdomains of the phases and the interface

$$
\begin{array}{ll}
N_{\mathrm{ph}}^{(\gamma)}(\mathbf{y})=0 \text { if } \mathbf{y} \notin \Theta^{(\gamma)} ; & \Theta^{(\gamma)} \subset \Theta \\
N_{\text {int }}^{(\beta)}(\mathbf{y})=0 \text { if } \mathbf{y} \notin S^{(\beta)} ; & S^{(\beta)} \subset S
\end{array}
$$

Employing Eqs. 24 and 25, $\tilde{\boldsymbol{\epsilon}}$ is expressed in terms of the damage induced strain and displacement jump coefficients:

$$
\tilde{\epsilon}_{i j}(\mathbf{x}, \mathbf{y}, t)=\sum_{\gamma} \tilde{P}_{i j k l}^{(\gamma)}(\mathbf{y}) \mu_{k l}^{(\gamma)}(\mathbf{x}, t)+\sum_{\beta} \tilde{R}_{i j p}^{(\beta)}(\mathbf{y}) \hat{\delta}_{p}^{(\beta)}(\mathbf{x}, t)
$$

in which, the coefficient tensors $\tilde{\mathbf{P}}$ and $\tilde{\mathbf{R}}$ are:

$$
\begin{aligned}
& \tilde{P}_{i j k l}^{(\gamma)}(\mathbf{y})=\int_{\Theta(\gamma)} g_{i j k l}^{\mathrm{ph}}(\mathbf{y}, \check{\mathbf{y}}) N_{\mathrm{ph}}^{(\gamma)}(\check{\mathbf{y}}) d \check{\mathbf{y}} \\
& \tilde{R}_{i j p}^{(\beta)}(\mathbf{y})=\int_{S^{(\beta)}} g_{i j q}^{\mathrm{int}}(\mathbf{y}, \check{\mathbf{y}}) N_{\mathrm{int}}^{(\beta)}(\check{\mathbf{y}}) \hat{\mathbf{e}}_{q}(\check{\mathbf{y}}) d \check{\mathbf{y}}
\end{aligned}
$$

where $\hat{\mathbf{e}}_{q}$ denotes the transformation vector between the global and local coordinate systems along the interface.

Substituting Eqs. 24 and 25 into Eq. 22, premultiplying the resulting equation with $N_{\mathrm{ph}}^{(\eta)}(\hat{\mathbf{y}})$, and integrating over the domain of the RVE yields $(\eta=1,2, \ldots, n)$ :

$$
\begin{aligned}
\sum_{\Delta=1}^{n}\left\{\left[1-\omega_{\mathrm{ph}}^{(\Delta)}(\mathbf{x}, t)\right] \int_{\Theta(\Delta)} N_{\mathrm{ph}}^{(\Delta)}(\mathbf{y}) \tilde{P}_{i j q r}^{(\eta)}(\mathbf{y}) L_{i j m n}(\mathbf{y})\right. & \\
{\left.\left[A_{m n k l}(\mathbf{y}) \bar{\epsilon}_{k l}(\mathbf{x}, t)+\tilde{\epsilon}_{m n}(\mathbf{x}, \mathbf{y}, t)\right] d \mathbf{y}\right\} } & =0
\end{aligned}
$$

Similarly, substituting Eqs. 24 and 25 into Eq. 23, premultiplying the resulting equation with $N_{\text {int }}^{(\alpha)}(\hat{\mathbf{y}})$ and integrating over the domain of the RVE yields $(\alpha=1,2, \ldots, m)$ :

$$
\begin{array}{r}
\sum_{\Delta=1}^{n}\left\{\left[1-\omega_{\mathrm{ph}}^{(\Delta)}(\mathbf{x}, t)\right] \int_{\Theta(\Delta)} N_{\mathrm{ph}}^{(\Delta)}(\mathbf{y}) \tilde{R}_{i j p}^{(\alpha)}(\mathbf{y}) L_{i j m n}(\mathbf{y})\right. \\
\left.\left[A_{m n k l}(\mathbf{y}) \bar{\epsilon}_{k l}(\mathbf{x}, t)+\tilde{\epsilon}_{m n}(\mathbf{x}, \mathbf{y}, t)\right] d \mathbf{y}\right\}=-\hat{t}_{p}^{(\alpha)}(\mathbf{x}, t)
\end{array}
$$


Substituting Eq. 28 into Eqs. 31 and 32 results in

$$
\begin{aligned}
& \sum_{\Delta=1}^{n}\left\{[ 1 - \omega _ { \mathrm { ph } } ^ { ( \Delta ) } ( \mathbf { x } , t ) ] \left[C_{i j k l}^{(\eta \Delta)} \bar{\epsilon}_{k l}(\mathbf{x}, t)+\sum_{\gamma=1}^{n} F_{i j k l}^{(\eta \Delta \gamma)} \mu_{k l}^{(\gamma)}(\mathbf{x}, t)\right.\right. \\
& \left.\left.+\sum_{\beta=1}^{m} J_{i j p}^{(\eta \Delta \beta)} \hat{\delta}_{p}^{(\beta)}(\mathbf{x}, t)\right]\right\}=0 \\
& \sum_{\Delta}\left\{[ 1 - \omega _ { \mathrm { ph } } ^ { ( \Delta ) } ( \mathbf { x } , t ) ] \left[D_{p k l}^{(\alpha \Delta)} \bar{\epsilon}_{k l}(\mathbf{x}, t)+\sum_{\gamma=1}^{n} J_{k l p}^{(\gamma \Delta \alpha)} \mu_{k l}^{(\gamma)}(\mathbf{x}, t)\right.\right. \\
& \left.\left.+\sum_{\beta=1}^{m} M_{p q}^{(\alpha \Delta \beta)} \hat{\delta}_{p}^{(\beta)}(\mathbf{x}, t)\right]\right\}=-\hat{t}_{p}^{(\alpha)}(\mathbf{x}, t)
\end{aligned}
$$

where,

$$
\begin{aligned}
C_{i j k l}^{(\eta \Delta)} & =\int_{\Theta(\Delta)} \tilde{P}_{q r i j}^{(\eta)}(\mathbf{y}) L_{q r m n}(\mathbf{y}) A_{m n k l}(\mathbf{y}) N_{\mathrm{ph}}^{(\Delta)}(\mathbf{y}) d \mathbf{y} \\
D_{p k l}^{(\alpha \Delta)} & =\int_{\Theta^{(\Delta)}} \tilde{R}_{i j p}^{(\alpha)}(\mathbf{y}) L_{i j m n}(\mathbf{y}) A_{m n k l}(\mathbf{y}) N_{\mathrm{ph}}^{(\Delta)}(\mathbf{y}) d \mathbf{y} \\
F_{i j k l}^{(\eta \Delta \gamma)} & =\int_{\Theta(\Delta)} \tilde{P}_{q r i j}^{(\eta)}(\mathbf{y}) L_{q r m n}(\mathbf{y}) \tilde{P}_{m n k l}^{(\gamma)}(\mathbf{y}) N_{\mathrm{ph}}^{(\Delta)}(\mathbf{y}) d \mathbf{y} \\
J_{i j p}^{(\eta \Delta \beta)} & =\int_{\Theta^{(\Delta)}} \tilde{P}_{q r i j}^{(\eta)}(\mathbf{y}) L_{q r m n}(\mathbf{y}) \tilde{R}_{m n p}^{(\beta)}(\mathbf{y}) N_{\mathrm{ph}}^{(\Delta)}(\mathbf{y}) d \mathbf{y} \\
M_{p q}^{(\alpha \Delta \beta)} & =\int_{\Theta^{(\Delta)}} \tilde{R}_{i j p}^{(\alpha)}(\mathbf{y}) L_{i j m n}(\mathbf{y}) \tilde{R}_{m n q}^{(\beta)}(\mathbf{y}) N_{\mathrm{ph}}^{(\Delta)}(\mathbf{y}) d \mathbf{y}
\end{aligned}
$$

in which, $\mathbf{C}^{(\eta \Delta)}, \mathbf{D}^{(\alpha \Delta)}, \mathbf{F}^{(\eta \Delta \gamma)}, \mathbf{J}^{(\eta \Delta \beta)}$ and $\mathbf{M}^{(\alpha \Delta \beta)}$ are coefficient tensors. The interface traction coefficient, $\hat{\mathbf{t}}^{(\alpha)}$ is:

$$
\hat{t}_{i}^{(\alpha)}(\mathbf{x}, t)=\int_{S^{(\alpha)}} N_{\text {int }}^{(\alpha)}(\mathbf{y}) \hat{t}_{i}(\mathbf{x}, \mathbf{y}, t) d \mathbf{y}
$$

The relationship between the interface traction and the displacement jump is nonlinear. It is, therefore, not possible to derive explicit expressions for the relationship between the traction and displacement jump coefficients. In this manuscript, the relationship between the pointwise tractions and displacement jumps are adopted to represent the relationship between the traction and displacement jump coefficients. This approach has also been employed in a number of previous investigations (e.g., [20]). The unilateral contact and adhesion conditions are expressed as

$$
\begin{gathered}
\delta^{N(\alpha)}(\mathbf{x}, t) \geq 0 \\
t^{N(\alpha)}(\mathbf{x}, t)-\left[1-\omega_{\text {int }}^{(\alpha)}(\mathbf{x}, t)\right] k_{N}^{(\alpha)} \delta^{N(\alpha)}(\mathbf{x}, t) \leq 0 \\
\left\{t^{N(\alpha)}(\mathbf{x}, t)-\left[1-\omega_{\text {int }}^{(\alpha)}(\mathbf{x}, t)\right] k_{N}^{(\alpha)} \delta^{N(\alpha)}(\mathbf{x}, t)\right\} \delta^{N(\alpha)}(\mathbf{x}, t)=0
\end{gathered}
$$

The tangential adhesion condition is also written in a similar form as

$$
t_{\rho}^{T(\alpha)}(\mathbf{x}, t)-\left[1-\omega_{\text {int }}^{(\alpha)}(\mathbf{x}, t)\right] k_{T}^{(\alpha)} \delta_{\rho}^{T(\alpha)}(\mathbf{x}, t)=0
$$


Similar to the interface traction-separation conditions, the nonlinear evolution of the phase and interface damage coefficients, $\omega_{\mathrm{ph}}^{(\gamma)}$ and $\omega_{\text {int }}^{(\alpha)}$, are expressed in terms of the field coefficients:

$$
\begin{gathered}
\omega_{\mathrm{ph}}=\omega_{\mathrm{ph}}\left(\sigma_{i j}, \epsilon_{i j}, q_{a}\right) \rightarrow \omega_{\mathrm{ph}}^{(\gamma)}=\omega_{\mathrm{ph}}^{(\gamma)}\left(\sigma_{i j}^{(\gamma)}, \epsilon_{i j}^{(\gamma)}, q_{a}^{(\gamma)}\right) \\
\omega_{\text {int }}=\omega_{\text {int }}\left(t_{i}, \delta_{i}, q_{a}^{\mathrm{int}}\right) \rightarrow \omega_{\text {int }}^{(\alpha)}=\omega_{\text {int }}^{(\alpha)}\left(t_{i}^{(\alpha)}, \delta_{i}^{(\alpha)}, q_{a}^{\operatorname{int}(\alpha)}\right)
\end{gathered}
$$

in which, $\mathbf{q}$ and $\mathbf{q}^{\text {int }}$ are state variables defining the evolution of the phase and interface damage variables, respectively, and;

$$
[\cdot]^{(\gamma)}(\mathbf{x}, t)=\int_{\Theta(\gamma)} N_{\mathrm{ph}}^{(\gamma)}(\mathbf{y})[\cdot](\mathbf{x}, \mathbf{y}, t) d \mathbf{y} ; \quad[\cdot]^{(\alpha)}(\mathbf{x}, t)=\int_{S^{(\alpha)}} N_{\text {int }}^{(\alpha)}(\mathbf{y})[\cdot](\mathbf{x}, \mathbf{y}, t) d \mathbf{y}
$$

Equilibrium equations (Eqs. 33 and 34), in addition to the interface conditions provided by Eqs 41-44, and the evolution equations for the phase and interface damage coefficients form the reduced order model. The reduced order model is solved to obtain the unknown coefficients $\boldsymbol{\mu}^{(\gamma)}$ and $\hat{\boldsymbol{\delta}}^{(\alpha)}$.

The macroscopic stress tensor is expressed in terms of $\boldsymbol{\mu}^{(\gamma)}, \hat{\boldsymbol{\delta}}^{(\alpha)}$, and the macroscopic strain tensor by substituting Eq. 17 and 18 into Eq. 11 and using Eqs. 24 and 25 to obtain

$$
\bar{\sigma}_{i j}(\mathbf{x}, t)=\sum_{\Delta}\left[1-\omega_{\mathrm{ph}}^{(\Delta)}(\mathbf{x}, t)\right]\left[\bar{L}_{i j k l}^{(\Delta)} \bar{\epsilon}_{k l}(\mathbf{x}, t)+\sum_{\gamma} \bar{P}_{i j k l}^{(\Delta \gamma)} \mu_{k l}^{(\gamma)}(\mathbf{x}, t)+\sum_{\beta} \bar{R}_{i j p}^{(\Delta \beta)} \hat{\delta}_{p}^{(\beta)}(\mathbf{x}, t)\right]
$$

where, the coefficient tensors $\overline{\mathbf{L}}^{(\Delta)}, \overline{\mathbf{P}}^{(\Delta \gamma)}$ and $\overline{\mathbf{R}}^{(\Delta \beta)}$ are expressed as

$$
\begin{aligned}
\bar{L}_{i j k l}^{(\Delta)} & =\frac{1}{|\Theta|} \int_{\Theta(\Delta)} N_{\mathrm{ph}}^{(\Delta)}(\mathbf{y}) L_{i j m n}(\mathbf{y}) A_{m n k l}(\mathbf{y}) d \mathbf{y} \\
\bar{P}_{i j k l}^{(\Delta \gamma)} & =\frac{1}{|\Theta|} \int_{\Theta(\Delta)} N_{\mathrm{ph}}^{(\Delta)}(\mathbf{y}) L_{i j m n}(\mathbf{y}) \tilde{P}_{m n k l}^{(\gamma)}(\mathbf{y}) d \mathbf{y} \\
\bar{R}_{i j p}^{(\Delta \beta)} & =\frac{1}{|\Theta|} \int_{\Theta(\Delta)} N_{\mathrm{ph}}^{(\Delta)}(\mathbf{y}) L_{i j m n}(\mathbf{y}) \tilde{R}_{m n p}^{(\beta)}(\mathbf{y}) d \mathbf{y}
\end{aligned}
$$

\section{Computational Aspects}

The implementation of the proposed reduced-order multiscale model is conducted in two stages. The preprocessing stage consists of determining the model order, partitioning of the RVE based upon the model order, and computing the coefficient tensors associated with the reduced order model. The macroscopic analysis stage consists of evaluating the macroscale problem described in Section 2.2 using a numerical method. In this study, the macroscopic analyses are conducted using the finite element method. The commercially available finite element analysis program, Abaqus, is employed. Reduced order multiscale models and direct numerical simulations for the verification of the proposed approach are conducted using the user supplied subroutine capabilities. The remainder of this section discusses a novel strategy for selection of the model order and partitioning of the RVE domain, as well as the numerical evaluation of the reduced order model. 


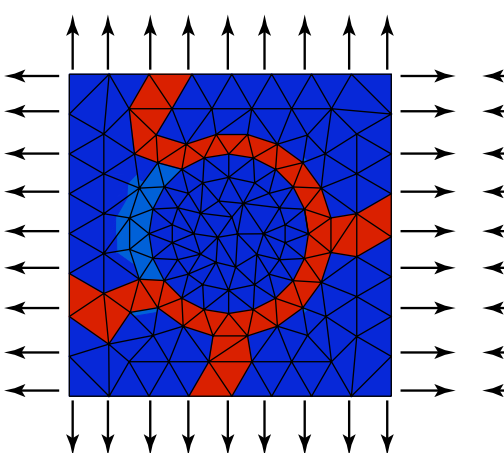

(a)

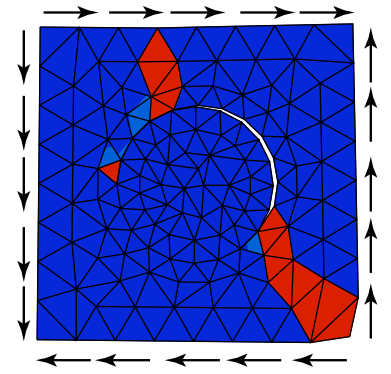

(d)

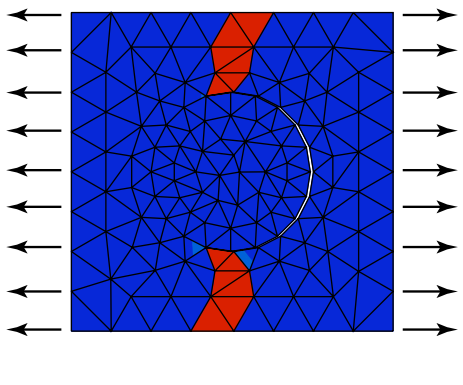

(b)

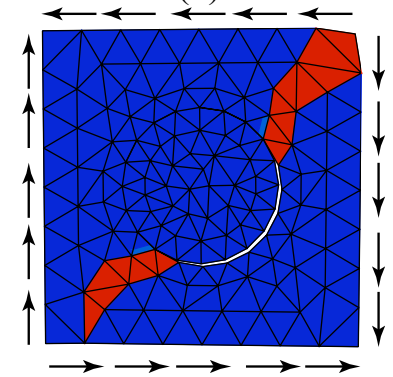

(e)

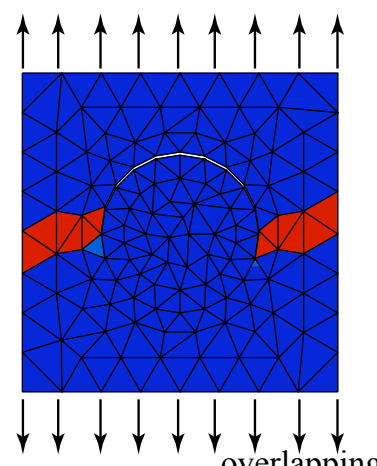

(c)

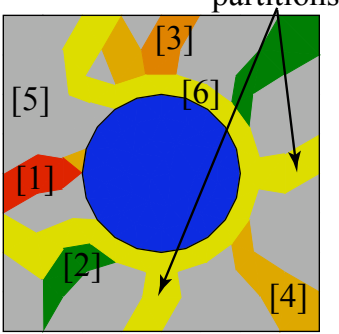

(f)

Figure 2: The partitioning and model reduction strategy. Failure profiles within the RVE subjected to (a) uniform biaxial loading; (b) uniaxial in the lateral direction; (c) uniaxial loading in the vertical direction; (d) shear loading along the positive direction; (e) shear loading along the negative direction; (f) resultant partitions.

\subsection{Reduced-Order Model Development Strategy}

The proper partitioning of the RVE domain is critical to the efficiency and the accuracy of the proposed reduced order modeling approach. The partitioning of the RVE consists of the selection of the number of phase $(n)$ and interface $(m)$ partitions, as well as the domain of each phase $\left(\Theta^{(\gamma)}\right)$ and interface $\left(S^{(\beta)}\right)$ partition. Theoretically, as the number of partitions, $n$ and $m$, increase, the accuracy of the reduced model increases at the expense of additional computational cost. The accuracy of the reduced order model is also strongly affected by the selection of the partition domains, $\Theta^{(\gamma)}$ and $S^{(\beta)}$ for a given number of phase and interface partitions. Previous and current investigations found significant variability in the performance of a reduced order model based on the partitioning strategy. It is possible to adaptively select the order of the reduced-order model based on a-priori error measures associated with the state of the failure processes during the macroscopic simulations. Such a dynamic partitioning strategy was proposed in Ref. [21]. The dynamic strategy resembles $h$-version adaptive finite element modeling with goal oriented mesh adaptivity [23], or multilevel-multiscale modeling, in which the heterogeneity within the process zones are adaptively resolved [24]. The dynamic partitioning strategy, while rigorous, comes with a significant increase in computational cost. The additional computational cost is due to error assessment and recomputation of the coefficient tensors during the macroscopic analysis.

In this manuscript, a novel static partitioning strategy is presented, in which, the RVE domain partitions and the model order is identified prior to the macroscopic analysis. The present approach provides a model selection strategy capable of accounting for the failure modes within 
the microstructure using a small number of domain partitions. The model selection strategy consists of identification of the failure paths within the microstructure when subjected to a number of loading modes, and partitioning the domain of the RVE as well as the interfaces by selecting each failure path as a partition. The failure paths within the microstructure are identified by conducting detailed RVE-level simulations. The RVE is subjected to uniform macroscopic strain modes (e.g., uniaxial tensile or compression and shear). Figure 2 illustrates the identification of the failure paths in a 2-D particle reinforced matrix under uniform macroscopic axial and shear strains. The failure path due to each loading condition is marked as an individual partition as shown in Fig. 2e. RVE-level simulations were conducted by applying Dirichlet conditions along the boundaries when determining the failure paths. This, along with the unstructured finite element mesh leads to unsymmetric failure paths despite symmetry in the RVE geometry. Periodic boundary conditions are maintained along the RVE boundaries in the multiscale model.

The failure paths associated with different loading modes intersect each other as demonstrated in Fig. 2. Hence, the phase partitions are allowed to overlap. The phase shape functions, are selected to accommodate such an overlap. Let the domain of the RVE be partitioned into $n$ possibly intersecting subdomains, denoted by $\Theta^{(\gamma)}, \gamma=1,2, \ldots, n$. The union of the subdomains spans the domain of the RVE:

$$
\Theta \equiv \bigcup_{\gamma=1}^{n} \Theta^{(\gamma)}
$$

The intersection between two partitions are denoted as $\Theta^{(\gamma \eta)} \equiv \Theta^{(\gamma)} \cap \Theta^{(\eta)}$. A material point within the RVE may lie in all $n$ partitions or less. The intersections between multiple partitions are defined by repetitive Greek superscripts: $\Theta^{(\gamma \eta \nu \ldots)} \equiv \Theta^{(\gamma)} \cap \Theta^{(\eta)} \cap \Theta^{(\nu)} \ldots$. The shape functions for the reduced order model, $N_{\mathrm{ph}}^{(\gamma)}$, are chosen as:

$$
N_{\mathrm{ph}}^{(\gamma)}(\mathbf{y})=\left\{\begin{array}{cc}
\frac{1}{i} & \text { if } \quad \mathbf{y} \in \Theta_{i}^{(\gamma)} \\
0 & \text { elsewhere }
\end{array}\right.
$$

in which, $i=1,2, \ldots, n$, and;

$$
\begin{aligned}
\Theta_{1}^{(\gamma)} & \equiv \Theta^{(\gamma)} \backslash \bigcup_{\eta=1}^{n} \Theta^{(\gamma \eta)} \\
\Theta_{2}^{(\gamma)} & \equiv \bigcup_{\eta=1}^{n}\left[\Theta^{(\gamma \eta)} \backslash \bigcup_{\nu=1}^{n} \Theta^{(\gamma \eta \nu)}\right]
\end{aligned}
$$

The expressions for $\Theta_{3}^{\gamma} \ldots \Theta_{n}^{\gamma}$ are derived analogously. The shape functions defined in Eq. 53 allow the possibility of intersecting shape functions, and satisfy the partition of unity property:

$$
\sum_{\gamma=1}^{n} N_{\mathrm{ph}}^{(\gamma)}(\mathbf{y})=1 ; \quad \mathbf{y} \in \Theta
$$

The interface shape functions are continuous across the partitions to satisfy the continuity of tractions and displacement jumps across the interface partitions. Consider the partitioning of the interface $S$ into $m$ overlapping subdomains $S^{(\alpha)}$. The interface shape function, $N_{\text {int }}^{(\alpha)}$, is 
a linear combinations of standard finite element shape functions corresponding to the nodes along the interface partition, $S^{(\alpha)}[21]$

$$
N_{\text {int }}^{(\alpha)}(\mathbf{y})=\sum_{a \in S^{(\alpha)}} N_{a}(\mathbf{y}) ; \quad \mathbf{y} \in S
$$

in which, $N_{a}$ is the standard finite element shape function associated with the microscopic finite element mesh node $a$.

\subsection{Numerical Evaluation of the Reduced-Order Model}

The evaluation of the reduced order model for the microscale problem constitutes the macroscopic stress update at a macroscopic point. The reduced order model is evaluated using the active set strategy to account for the contact conditions at the interfaces.

Given: At a macroscopic material point $\mathbf{x}$ and at time $t$, the equilibrium state defined by the macroscopic strain tensor ${ }_{t} \overline{\boldsymbol{\epsilon}}$; the inelastic strain and displacement jump coefficients, ${ }_{t} \boldsymbol{\mu}^{(\gamma)}$ and ${ }_{t} \hat{\boldsymbol{\delta}}^{(\alpha)}$, respectively, where $\gamma=1,2, \ldots, n$ and $\alpha=1,2, \ldots, m$; state variables ${ }_{t} \mathbf{q}^{(\gamma)}$ and ${ }_{t} \mathbf{q}_{\text {int }}{ }^{(\alpha)}$, which define the evolution of the phase and interface damage state, ${ }_{t} \omega_{\mathrm{ph}}^{(\gamma)}$ and ${ }_{t} \omega_{\text {int }}^{(\alpha)}$, respectively; as well as the change in the macroscopic strain state, $\Delta \overline{\boldsymbol{\epsilon}}$ (taking an assumed strain approach in the numerical evaluation of the macroscopic boundary value problem).

Compute: The current values (at time: $t+\Delta t$ ) of the inelastic strain and displacement coefficients, $\boldsymbol{\mu}^{(\gamma)}$ and $\hat{\boldsymbol{\delta}}^{(\alpha)}$, respectively; the current damage state, $\omega_{\mathrm{ph}}^{(\gamma)}$ and $\omega_{\text {int }}^{(\alpha)}$; state variables, $\mathbf{q}^{(\gamma)}$ and $\mathbf{q}_{\text {int }}{ }^{(\alpha)}$ and the macroscopic stress, $\overline{\boldsymbol{\sigma}}$.

In this section, we will employ vector notation using the classical index contractions (e.g., $\left.\left.\mathbf{L}=\left\{L_{I J}\right\}\right) \leftarrow\left\{L_{i j k l}\right\}\right)$. A left subscript $t$ indicates the value of the function at time $t$. A left superscript denotes iteration count. The eigendeformation vector is defined as:

$$
\mathbf{d}=\left\{\boldsymbol{\mu}^{(1)}, \ldots, \boldsymbol{\mu}^{(n)}, \hat{\boldsymbol{\delta}}^{(1)}, \ldots, \hat{\boldsymbol{\delta}}^{(m)}\right\}^{T}
$$

The active set is defined as the set of all interface partitions in which normal displacement jump coefficients are zero:

$$
\mathcal{A}=\left\{\alpha \mid \alpha \in\{1, \ldots, m\} ; \delta^{N(\alpha)}=0\right\}
$$

We define the discrete system of nonlinear equations, $\Psi$, based on reduced order model as:

$$
\Psi(\mathbf{d})=\mathbf{K}\left(\omega_{\mathrm{ph}}^{(\gamma)}, \omega_{\mathrm{int}}^{(\alpha)}\right) \mathbf{d}+\mathbf{f}\left(\omega_{\mathrm{ph}}^{(\gamma)}\right)
$$

in which,

$$
\mathbf{K}\left(\omega_{\mathrm{ph}}^{(\gamma)}, \omega_{\text {int }}^{(\alpha)}\right)=\mathbf{K}_{\mathbf{t}}\left(\omega_{\text {int }}^{(\alpha)}\right)+\sum_{\Delta=1}^{n}\left[1-\omega_{\mathrm{ph}}^{(\Delta)}\right] \mathbf{K}^{(\Delta)}
$$

and,

$$
\mathbf{K}^{(\Delta)}=\left[\begin{array}{cccccc}
\mathbf{F}^{(1 \Delta 1)} & \cdots & \mathbf{F}^{(1 \Delta n)} & \mathbf{G}^{(1 \Delta 1)} & \cdots & \mathbf{G}^{(1 \Delta m)} \\
\vdots & \ddots & \vdots & \vdots & \ddots & \vdots \\
\mathbf{F}^{(n \Delta 1)} & \cdots & \mathbf{F}^{(n \Delta n)} & \mathbf{G}^{(n \Delta 1)} & \cdots & \mathbf{G}^{(n \Delta m)} \\
\hat{\mathbf{G}}^{(1 \Delta 1)} & \cdots & \hat{\mathbf{G}}^{(1 \Delta n)} & \mathbf{H}^{(1 \Delta 1)} & \cdots & \mathbf{H}^{(1 \Delta m)} \\
\vdots & \ddots & \vdots & \vdots & \ddots & \vdots \\
\hat{\mathbf{G}}^{(m \Delta 1)} & \cdots & \hat{\mathbf{G}}^{(m \Delta n)} & \mathbf{H}^{(m \Delta 1)} & \cdots & \mathbf{H}^{(m \Delta m)}
\end{array}\right]
$$


$\mathbf{K}^{(\Delta)}$ are symmetric matrices since $\hat{\mathbf{G}}^{(\alpha \Delta \eta)}=\mathbf{G}^{T(\eta \Delta \alpha)}, \mathbf{F}^{(\eta \Delta \gamma)}=\mathbf{F}^{T(\gamma \Delta \eta)}$ and $\mathbf{H}^{(\alpha \Delta \beta)}=$ $\mathbf{H}^{T(\beta \Delta \alpha)}$. $K_{\text {trac }}^{t}$ is defined as

$$
\mathbf{K}_{\mathbf{t}}=\left[\begin{array}{cccccc}
0 & \cdots & 0 & 0 & \cdots & 0 \\
\vdots & \ddots & \vdots & \vdots & \ddots & \vdots \\
0 & \cdots & 0 & 0 & \cdots & 0 \\
0 & \cdots & 0 & \hat{\mathbf{k}}^{(1)} & \cdots & 0 \\
\vdots & \ddots & \vdots & \vdots & \ddots & \vdots \\
0 & \cdots & 0 & 0 & \cdots & \hat{\mathbf{k}}^{(m)}
\end{array}\right] ; \quad \hat{\mathbf{k}}^{(\alpha)}=\left(1-\omega_{\text {int }}^{(\alpha)}\right)\left[\begin{array}{ccc}
k_{N}^{(\alpha)} & 0 & 0 \\
0 & k_{T}^{(\alpha)} & 0 \\
0 & 0 & k_{T}^{(\alpha)}
\end{array}\right]
$$

The symmetry of $\mathbf{K}$ leads to this formulation being denoted as a symmetric formulation. The solution of $\Psi=0$ is evaluated by symmetric nonlinear solvers rather than unsymmetric ones as has been the case in previous formulations. $\mathbf{f}$ is defined as

$$
\mathbf{f}\left(\omega_{\mathrm{ph}}^{(\gamma)}\right)=\sum_{\Delta=1}^{n}\left[1-\omega_{\mathrm{ph}}^{(\Delta)}\right] \mathbf{f}^{(\Delta)}
$$

and,

$$
\mathbf{f}^{(\Delta)}=\left\{\mathbf{C}^{(1 \Delta)}, \ldots, \mathbf{C}^{(n \Delta)}, \mathbf{D}^{(1 \Delta)}, \ldots, \mathbf{D}^{(m \Delta)}\right\}^{T} \overline{\boldsymbol{\epsilon}}
$$

In the case of tensile loading throughout the interface, the active set is empty $(\mathcal{A}=\emptyset)$, $\Psi(\mathbf{d})=0$ solves the reduced order model. When $\mathcal{A} \neq \emptyset$ a reduced system of equations is defined:

$$
\Psi_{\mathcal{A}}(\mathbf{d})=\mathbf{K}_{\mathcal{A}} \mathbf{d}_{\mathcal{A}}+\mathbf{f}_{\mathcal{A}}
$$

in which, $\mathbf{d}_{\mathcal{A}}$ and $\mathbf{f}_{\mathcal{A}}$ is constructed by removing each row which corresponds to $\delta_{N}^{(\alpha)}$ for each partition $\alpha$ in $\mathcal{A}$, from $\mathbf{d}$ and $\mathbf{f}$, respectively. $\mathbf{K}_{\mathcal{A}}$ is constructed by removing each row and column, which corresponds to $\delta_{N}^{(\alpha)}$ for each partition $\alpha$ in $\mathcal{A}$, from $\mathbf{K}$. The rows removed from Eq. 60 form:

$$
\Psi_{\overline{\mathcal{A}}}(\mathbf{d})=\mathbf{K}_{\overline{\mathcal{A}}} \mathbf{d}_{\overline{\mathcal{A}}}+\mathbf{f}_{\overline{\mathcal{A}}}
$$

The reduced order model is evaluated by ensuring $\Psi_{\mathcal{A}}(\mathbf{d})=0$ and $\Psi_{\overline{\mathcal{A}}}(\mathbf{d}) \geq 0$ are satisfied. The latter condition is necessary to ensure negative tractions upon compressive loading along the interfaces. The computational algorithm to evaluate the reduced order problem based on the active set strategy is provided in Box 1 . The algorithm is initiated by setting the working set, which approximates the active set at $(t+\Delta t)$, to the active set at time $t$, as well as setting the eigendeformation vector to ${ }_{t} \mathbf{d}$ (Step 1). Within each iteration $k$, the test eigendeformation vector, ${ }^{k} \hat{\mathbf{d}}$ is evaluated by a standard nonlinear root finding algorithm, such as Newton-Raphson or quasi-Newton methods (Step 2a). In this manuscript, a quasi-Newton SR1 method [25] is employed to compute the roots of $\Psi_{k} \mathcal{W}$. In this method, a symmetric-rank-one matrix is added to an approximation of the Jacobian of $\Psi_{k} \mathcal{W}$ at each iteration of the nonlinear solver. In practice, this update has been shown to provide very good approximations of the Jacobian resulting in superlinear convergence. The advantages of quasi-Newton methods are that they do not require an explicit formula for the Jacobian and that the update can be performed on the inverse Jacobian alleviating the need to solve a linear system of equations at each iteration. In this paper, the algorithm is initialized with a finite difference approximation to the Jacobian. If the computed normal displacement jump coefficients violate the impenetrability condition (Steps $2 \mathrm{~b}-\mathrm{c}$ ), the partition with the most severe violation is added to the working set. 
1. Initialize the algorithm by setting the initial guess for the eigendeformation vector and for the active set:

$$
k=1 ; \quad{ }^{0} \mathbf{d}={ }_{t} \mathbf{d} ;{ }^{1} \mathcal{W}={ }_{t} \mathcal{A}
$$

in which, ${ }^{k} \mathcal{W}$ denotes the working set. The working set is an approximation to the active set at iteration $k$.

2. Loop over the iterations $k$ :

(a) Compute ${ }^{k} \hat{\mathbf{d}}$ by evaluating $\Psi_{{ } \mathcal{W}}(\hat{\mathbf{d}})=0$ using a standard nonlinear root finding algorithm.

(b) Loop over each interface partition, $\alpha$, which is not in the working set (i.e., $\alpha \notin^{k} \mathcal{W}$ ):

i. If the impenetrability condition at partition $\alpha$ is violated (i.e., ${ }^{k} \hat{\delta}_{N}^{(\alpha)}<0$ ), compute the step size $0<\lambda^{(\alpha)}<1$ for each interface partition violating the impenetrability condition as:

$$
\lambda^{(\alpha)}=\frac{{ }^{k-1} \delta_{N}^{(\alpha)}}{k_{\hat{\delta}_{N}^{(\alpha)}-{ }^{k-1} \delta_{N}^{(\alpha)}}}
$$

(c) If the step size is reduced (i.e., $\left\{\lambda^{(\alpha)}\right\} \neq \emptyset$ ):

i. Compute $\beta=\arg \min \left\{\lambda^{(\alpha)}\right\}$

ii. Update the working set: ${ }^{k+1} \mathcal{W}={ }^{k} \mathcal{W} \cup\{\beta\}$

iii. $k \leftarrow k+1$

iv. ${ }^{k} \mathbf{d}=\lambda^{(\beta)}\left({ }^{k} \hat{\mathbf{d}}-{ }^{k-1} \mathbf{d}\right)+{ }^{k-1} \mathbf{d}$

v. Return to the beginning of the iteration loop

(d) Check if the unilateral conditions are violated in any partition within the working set: (i.e., If any component of $\Psi_{k} \overline{\mathcal{W}}(\mathbf{d})<0$ )

i. Compute $\beta=\arg \min \left(\Psi_{k} \overline{\mathcal{W}}\right)$

ii. Update the working set: ${ }^{k+1} \mathcal{W}={ }^{k} \mathcal{W}-\{\beta\}$,

iii. $k \leftarrow k+1$

iv. ${ }^{k} \mathbf{d}={ }^{k} \hat{\mathbf{d}}$

v. Return to the beginning of the iteration loop

(e) Update the eigendeformation vector: $\mathbf{d}={ }^{k} \mathbf{d}$

(f) Update the active set: $\mathcal{A}={ }^{k} \mathcal{W}$

(g) Exit the algorithm

End iteration loop

Box 1: The active set algorithm for evaluation of the reduced order model with unilateral contact constraints.

When the computed normal displacement jump coefficients do not violate the impenetrability condition, the unilateral contact constraints are checked within the active set. If the unilateral contact conditions are violated (i.e., if the computed interface traction coefficients are positive 
at partitions within the working set), the partition with the most severe violation (largest positive interface traction coefficient) is removed from the active set (Step 2d). When the unilateral contact constraints are satisfied, the active set, the eigendeformation vector, the associated internal state variables, and damage variables are updated (Steps 2e-g).

\subsection{Two-Order Reduced Modeling}

Reduced-order models fail to accurately capture the post-failure response of the representative volume element. The failure is defined as the loss of load carrying capacity along at least one loading direction. For instance, full damage within any one of the failure paths along with interface debonding in the RVE illustrated in Fig. 2 cause failure along the associated load direction. The reduced order models exhibit spurious residual stiffness upon failure, which prohibits proper redistribution of the stresses at the macroscopic scale. While increasing the model order diminishes the spurious residual stiffness, this approach increases the computational cost.

We propose a two-order modeling scheme to eliminate the residual-stress fields upon failure without significantly compromising the computational efficiency. In this approach, the stresses are computed based on a high order model, whereas the damage coefficients are evaluated using the low-order reduced-order model described in Section 4.1. The stress-update procedure for the two-order reduced model is as follows:

1. Evaluate the eigendeformation vector, $\mathbf{d}_{\text {low }}$, and damage coefficients, $\omega_{\mathrm{ph}}^{(\gamma)} ; \gamma=1,2, \ldots, n_{\text {low }}$ and $\omega_{\text {int }}^{(\alpha)} ; \alpha=1,2, \ldots, m_{\text {low }}$ for the low-order model using the numerical procedure described in Section 4.2. $n_{\text {low }}$ and $m_{\text {low }}$ are the orders for the low-order model selected by the reduced-order model development strategy described in Section 4.1.

2. Map the damage coefficients of the low-order model onto the high-order model partitions. The mapping of the damage coefficients onto the high-order model partitions is trivial when the high-order model is constructed by hierarchical subpartitioning of the low-order model. In this study, each finite element within the RVE domain constitutes a partition for the high-order model.

3. Evaluate the eigendeformation vector, $\mathbf{d}_{\text {high }}$, for the high order model by solving the linear system:

$$
\mathbf{d}_{\text {high }}=\mathbf{K}_{\text {high }}^{-1} \mathbf{f}_{\text {high }}
$$

4. Compute macroscopic stress (Eq. 48), using the eigendeformation vector of the high-order model.

\section{Numerical Verification}

The capabilities of the proposed reduced order modeling methodology are verified against direct finite element simulations. The verification study consists of (1) analysis of an RVE response and assessment of the reduced order model predictions, and (2) a three-point bending problem to assess the capabilities of the reduced order model in capturing the overall failure response of macroscopic structures.

\subsection{RVE Analysis}

The multiscale methodology described in the previous sections is applied to develop a mesomechanical model for a 2-D composite matrix with a circular inclusion. Figure 2 illustrates 


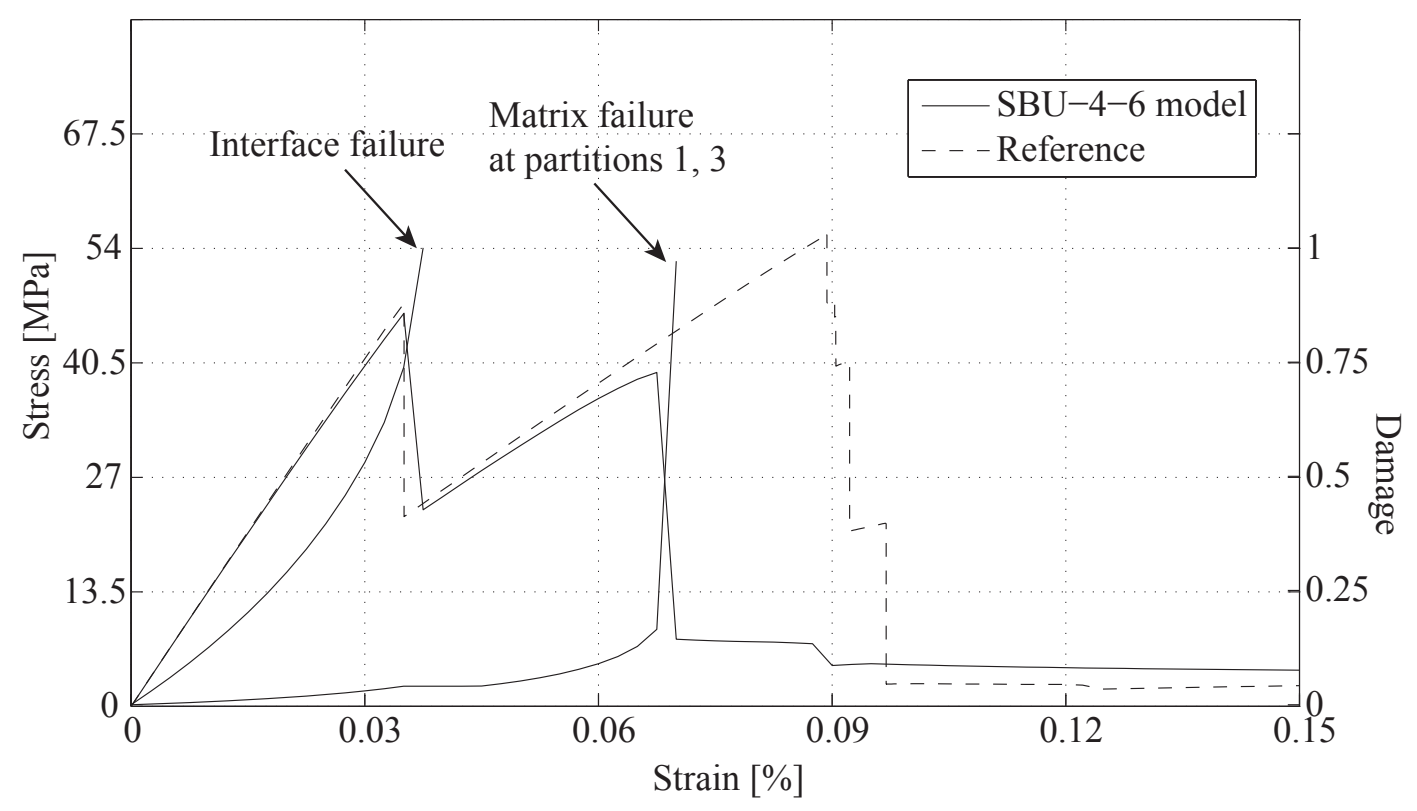

Figure 3: Stress-strain response and damage evolution within the RVE when subjected to uniform biaxial loading.

the geometry of the microstructure. The evolution of damage within the matrix and along the interface is modeled based on continuous damage mechanics models proposed in [21] for brittle composite constituents. The reinforcement is assumed to behave elastically within the range of applied loads.

The capabilities of the proposed multiscale model in capturing the failure modes for a range of loading conditions are verified by comparing the model simulations to direct numerical simulation of the representative volume element. The finite element mesh employed in these simulations is shown in Fig. 2. The characteristic material length scale associated with the matrix constituent is assumed to be $1 / 8$ of the RVE size. The finite element mesh of the RVE is designed to have an average size of $1 / 8$ of the RVE length scale to avoid numerical errors associated with mesh-sensitivity. The multiscale model is developed based on the reduced order model development strategy described in Section 4.1. The reduced order model is developed using the biaxial tension, uniaxial tension and shear loading modes. The partitioning of the reduced order model is shown in Fig. 2. The matrix phase and the interface are modeled using 6 and 4 partitions, respectively. This model is referred to as SBU-4-6 in the remainder of this manuscript.

The performance of model SBU-4-6 is compared to the results of the direct numerical simulations for the biaxial, uniaxial and shear loading cases. The force displacement diagrams in addition to the damage evolution in the interface and phase partitions are shown in Figs. 35. In biaxial loading, the failure along the interface is uniform and precedes the failure within the matrix phase. Upon interface debonding, the failure within the matrix propagates in the vertical and lateral directions. The evolution of damage within the matrix partitions and the interface clearly show that the failure modes are accurately captured by SBU-4-6. A similar trend is observed in model predictions when subjected to uniaxial (Fig. 5) and shear (Fig. 4) loading conditions. The failure mechanisms are captured with good accuracy when compared to the reference direct numerical simulations of the RVE. 


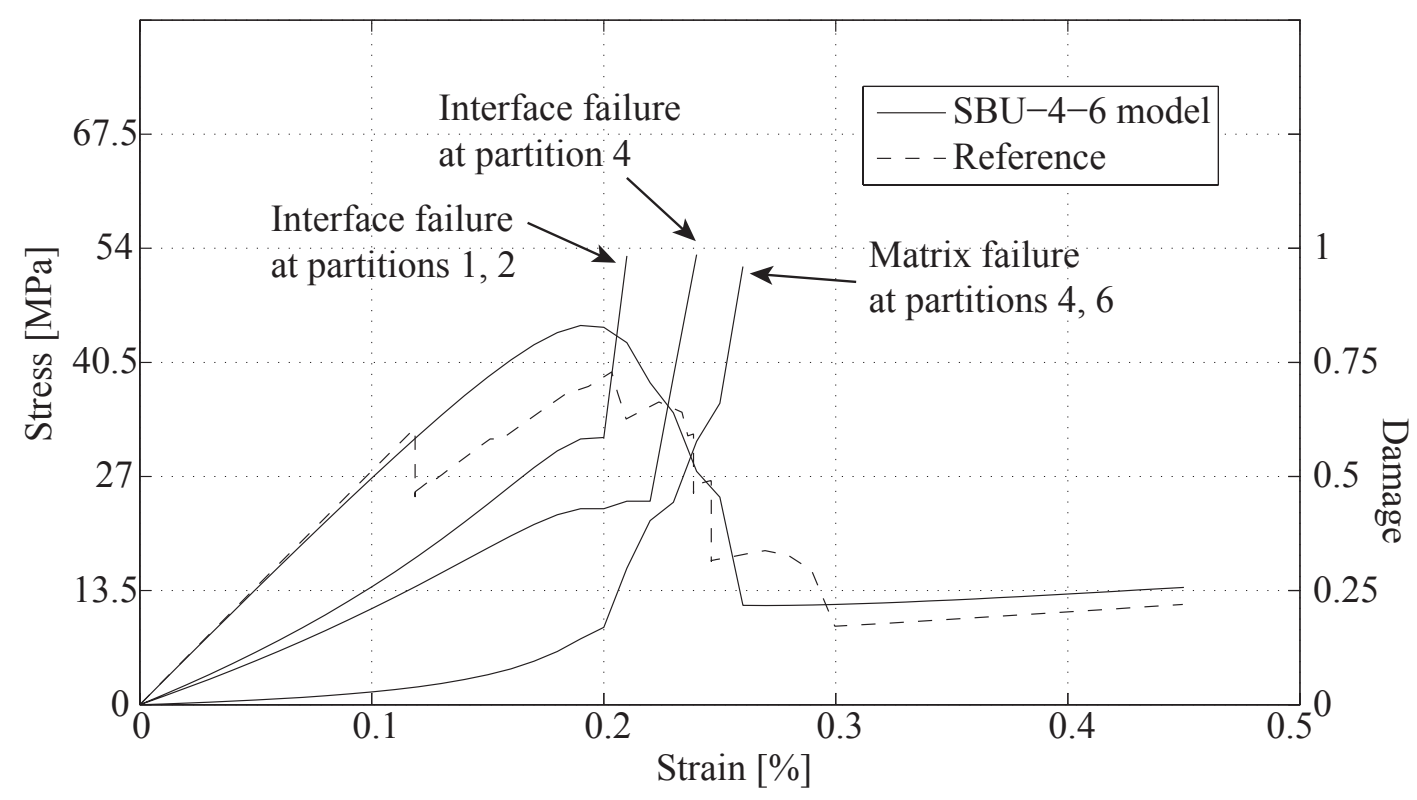

Figure 4: Stress-strain response and damage evolution within the RVE when subjected to shear loading.

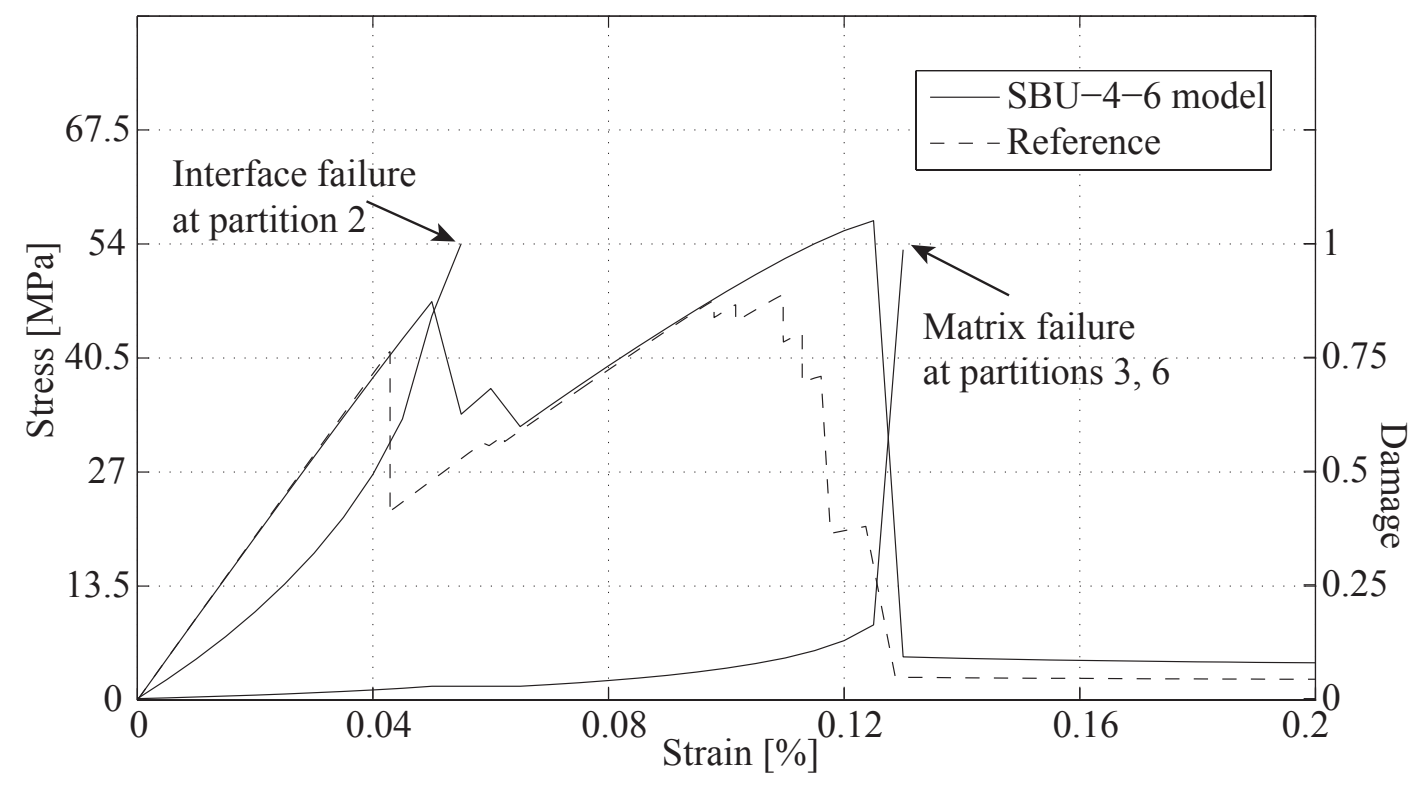

Figure 5: Stress-strain response and damage evolution within the RVE when subjected to uniaxial tensile loading in the lateral direction. 


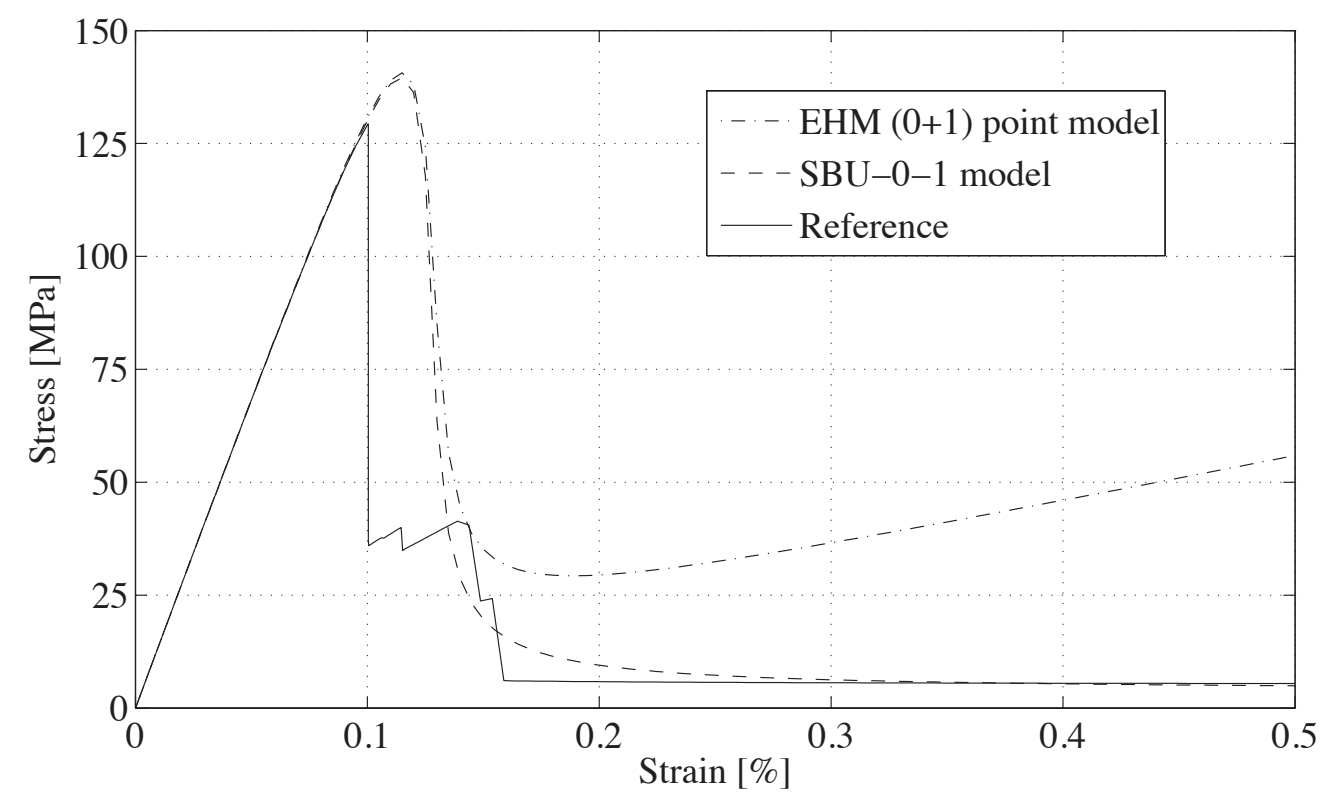

Figure 6: Stress-strain response when subjected to uniform biaxial tensile loading. The post-failure spurious residual stresses are eliminated with the proposed reduced order model.

Figure 6 illustrates the capability of the proposed reduced-order model in eliminating the spurious residual stresses in the post-failure regime. The spurious residual stresses present due to the modeling errors associated with reduction of the model order typically pollute the post-failure stress fields in the macroscopic analyses, since this effect partially constrains stress redistribution. Figure 6 compares the predictions of the proposed model along with the predictions of an eigendeformation-based homogenization model $(\mathrm{EHM}(0+1)$ point model) proposed in Ref. [21] along with the direct numerical simulations when subjected to uniform biaxial tension. The matrix-reinforcement interface is assumed to remain continuously bonded throughout the simulation. A 1-partition reduced order model, SBU-0-1, is adopted. The predictions of the EHM $(0+1)$ point model clearly demonstrate a residual strength upon failure of the matrix partition, while SBU-0-1 eliminates the spurious residual stresses.

The values of the model parameters used in the reduced order model are different than those of the direct numerical simulations. The objective of the proposed reduced order model is to capture the failure mechanisms within the heterogeneous material in a computationally efficient and accurate manner. The simulations conducted in this section demonstrate that the main failure mechanisms are captured with reasonable accuracy with the reduced order model. The model parameters for the reduced order model are computed by minimizing the discrepancy between the ultimate strength predicted by the proposed reduced order model and the direct numerical simulations in a least square sense. From the validation perspective, the reduced order model can be adopted to predict the response of heterogeneous systems by calibrating the material parameters of the damage models directly, based on experimental observations. A general discussion and methodologies for calibration and validation procedures for multiscale models are discussed in Ref. [26]. 


\subsection{Crack Propagation in a Beam Subjected to Three-Point Bending}

We consider a three-point bending of a cracked composite plate. The predictions of the SBU4-6 model are compared to a fine scale finite element model, which consists of 256 RVEs described in Section 5.1. The macroscale mesh for the multiscale model consists of 2564 -noded quadrilaterals. The volume fraction of the circular inclusions is $30 \%$. The circular inclusions are assumed to be isotropic and linear elastic with $E=200 \mathrm{GPa}$ and $\nu=0.3$. Damage processes are considered within the central third, and the matrix material is assumed to be linear elastic in the remainder of the plate. The elastic properties of the matrix material are $E=60 \mathrm{GPa}$ and $\nu=0.3$. The initial vertical matrix crack is assumed to extend $1 / 8$ th of the plate width.

Figures $7 \mathrm{a}$ and $7 \mathrm{~b}$ illustrates the propagation of the initial matrix crack and damage within the matrix as predicted by the direct numerical simulation and the SBU-4-6 model. In these simulations, the interface between the matrix and the inclusions are assumed to remain fully bonded for the duration of the loading. The propagation of the initial crack is arrested approximately halfway through the plate thickness when shear cracks develop at the edges of the applied loading. Figure 7 a shows the damage state within the third phase partition depicted in Fig. 2b. A comparison of the reaction force-applied displacement curves of the numerical simulation and the proposed multiscale model is shown in Fig. 8. The reduced-order model slightly over-predicts the strength of the composite plate. The errors associated with the SBU-4-6 are due to the blunting of the response fields across the failure paths within the microstructure by the model-reduction methodology. SBU-4-6 successfully captures the propagation and arrest of the initial crack and subsequent shear crack formation with reasonable accuracy.

Figures 10a and 10b shows the failure of the three-point bending plate in the presence of interface effects. The direct numerical simulation with the fine mesh shows that the path of crack propagation is significantly altered when the inclusion-matrix debonding is considered. The path of crack propagation displays a more jagged pattern with interaction between the matrix and interface cracks. Figure 10a displays the state of interface damage across the macroscale. The extent of interface damage is predicted by the SBU-4-6 model with reasonable accuracy. The comparison of the applied force-deflection curve predicted with the proposed multiscale model and the direct numerical simulations is shown in Fig. 9. The degradation effect of interface debonding on the overall performance of the plate is predicted by the proposed multiscale model with good accuracy.

\section{Conclusions}

In this manuscript, a reduced order multiscale computational methodology for failure analysis of heterogeneous materials is presented. The proposed approach provides a novel model development strategy for reduced-order models capable of efficiently and accurately representing the failure modes within the microstructure without recourse to detailed finite element modeling of the RVE. A two-order modeling approach is devised to eliminate spurious residual stresses upon failure, allowing accurate stress-redistribution within a macroscopic component. The resulting reduced-order model possesses symmetry, allowing efficient numerical evaluation of the microscale problem. The reduced order model proposed in this study is verified against direct numerical simulations. The proposed model captures the failure modes within the microstructure with good accuracy.

Perhaps, one of the more significant remaining challenge in multiscale computational mod- 


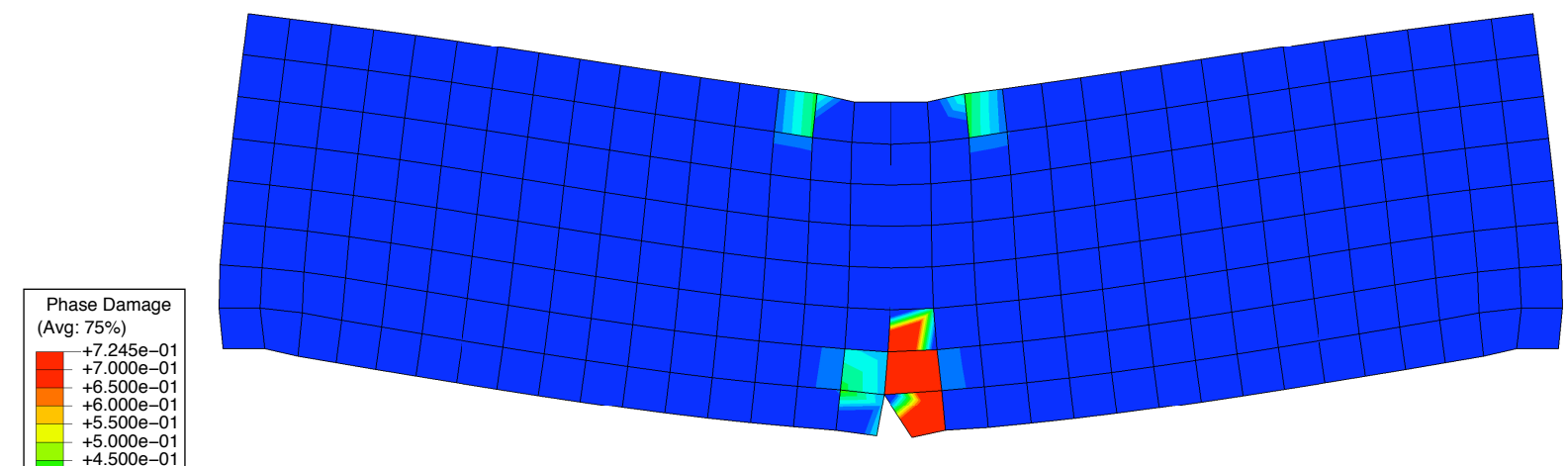

(a)

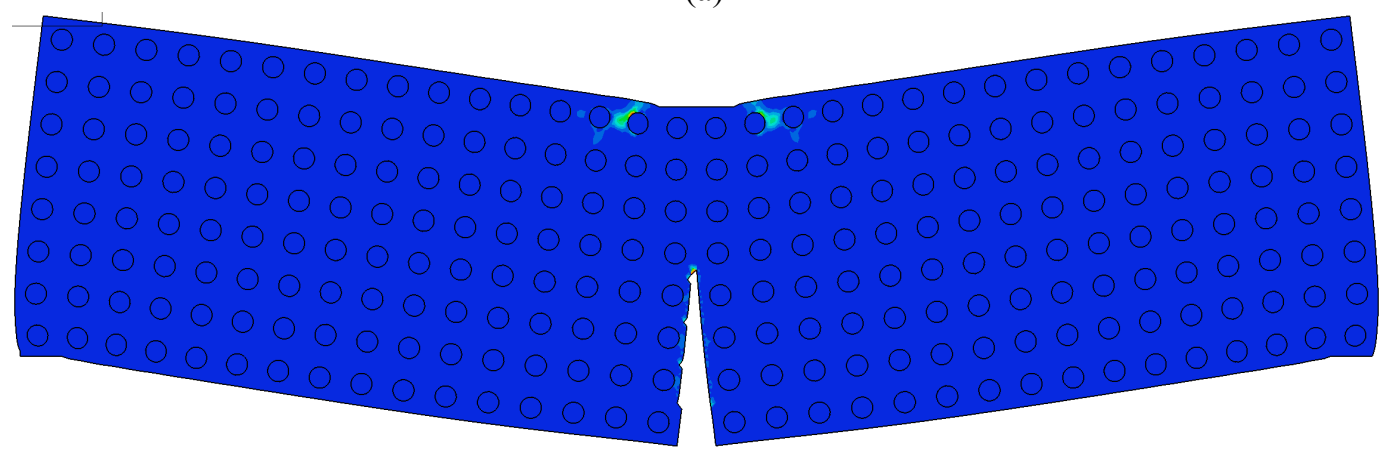

(b)

Figure 7: Damage profile of the 3-point bending beam specimen at the onset of shear fracture in the absence of interface debonding effects: (a) The prediction of the SBU-4-6 model. The contour represents the state of damage at phase partition 3; (b) Damage distribution predicted by the direct numerical simulation.

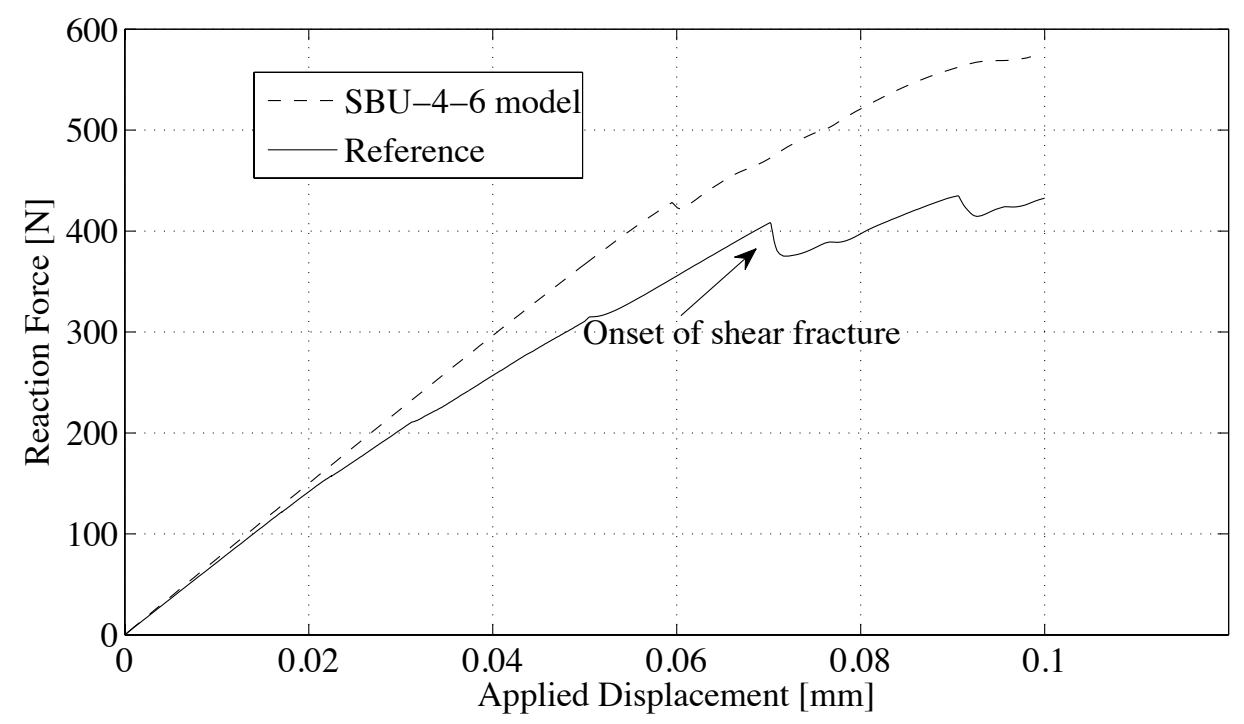

Figure 8: Comparison of the load-deflection curve between the multiscale SBU-4-6 model and the direct numerical simulation in the absence of interface debonding effects. 


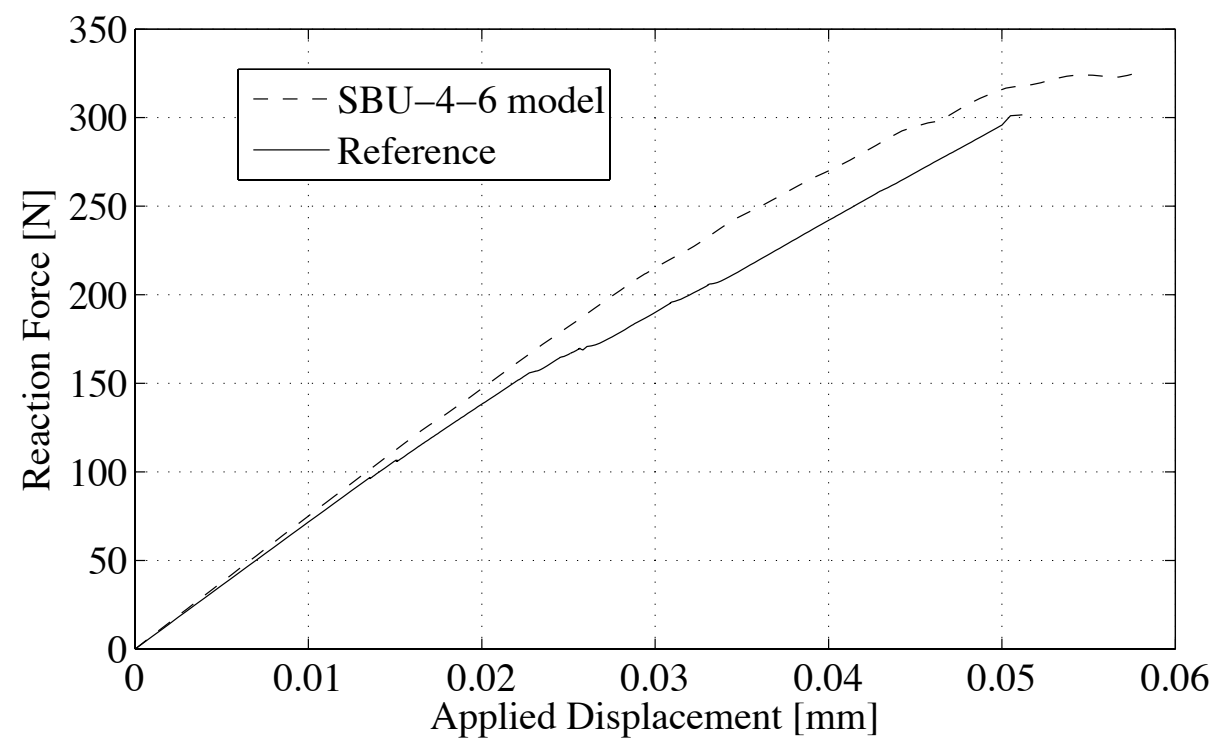

Figure 9: Comparison of the load-deflection curve between the multiscale SBU-4-6 model and the direct numerical simulation in the presence of interface debonding effects.
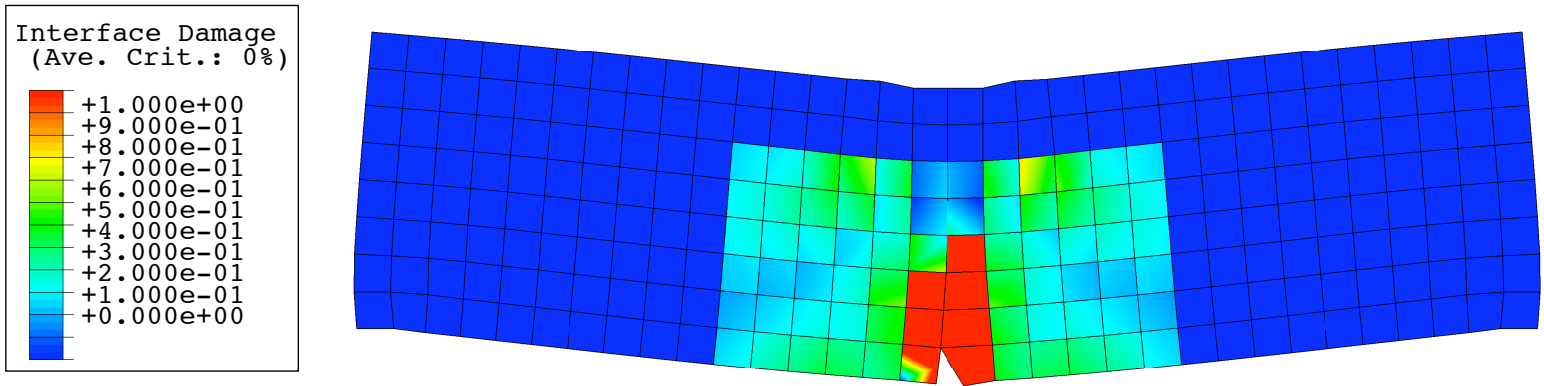

(a)

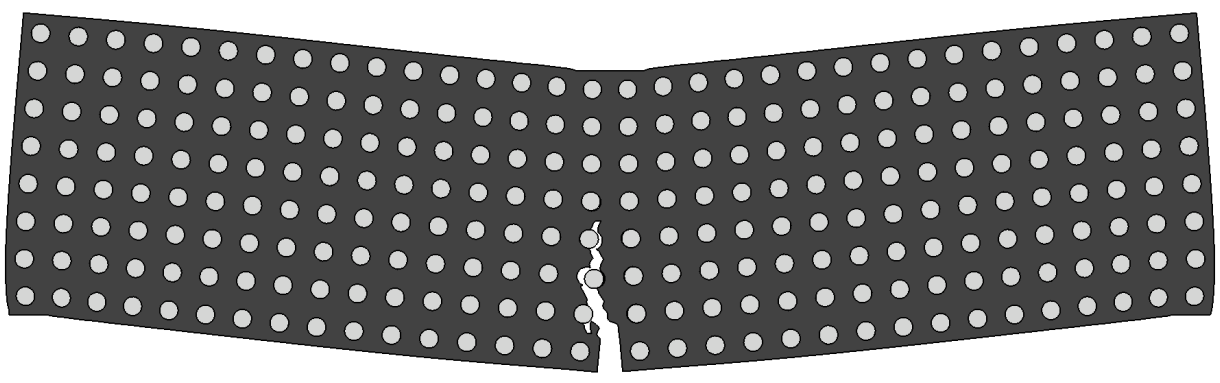

(b)

Figure 10: The deformed configuration of the 3-point bending beam specimen in the presence of interface debonding effects: (a) The prediction of the SBU-4-6 model. The contour represents the state of interface damage; (b) Crack profile predicted by the direct numerical simulation. 
eling of failure in heterogeneous materials is spurious mesh dependency of the resulting homogenized macroscale problem. While, the proposed reduced order modeling strategy eliminates mesh-dependency in the microscopic domain, the homogenized macroscale problem remains local. The localization limiters that eliminate spurious mesh dependency have been extensively investigated in the computational mechanics literature for homogeneous materials. In contrast, mesh dependency studies of multiscale material systems have been relatively limited. A rigorous, mesh independent, reduced order multiscale computational framework will be investigated in future studies.

\section{Acknowledgments}

The faculty start-up funds provided by Vanderbilt University are gratefully acknowledged.

\section{References}

[1] I. Babuska. Homogenization and application. mathematical and computational problems. In B. Hubbard, editor, Numerical Solution of Partial Differential Equations - III, SYNSPADE. Academic Press, 1975.

[2] A. Benssousan, J. L. Lions, and G. Papanicolaou. Asymptotic Analysis for Periodic Structures. North-Holland, Amsterdam, 1978.

[3] E. Sanchez-Palencia. Non-homogeneous media and vibration theory, volume 127 of Lecture notes in physics. Springer-Verlag, Berlin, 1980.

[4] P. M. Suquet. Elements of homogenization for inelastic solid mechanics. In E. SanchezPalencia and A. Zaoui, editors, Homogenization Techniques for Composite Media. Springer-Verlag, 1987.

[5] J. M. Guedes and N. Kikuchi. Preprocessing and postprocessing for materials based on the homogenization method with adaptive finite element methods. Comput. Meth. Appl. Mech. Engng., 83:143-198, 1990.

[6] O. van der Sluis, P.J.G. Schreurs, W. A. M.AM Brekelmans, and H.E.H. Meijer. Overall behaviour of heterogeneous elastoviscoplastic materials: effect of microstructural modelling. Mech. Mater., 32:449-462, 2000.

[7] J. Fish and R. Fan. Mathematical homogenization of nonperiodic heterogeneous media subjected to large deformation transient loading. Int. J. Numer. Meth. Engng., 76:10441064, 2008.

[8] M. G. D. Geers, V. Kouznetsova, and W. A. M. Brekelmans. Gradient-enhanced computational homogenization for the micro-macro scale transition. J. Phys. IV, 11:145-152, 2001.

[9] T. Belytschko, S. Loehnert, and J.-H. Song. Multiscale aggregating discontinuities: A method for circumventing loss of material stability. Int. J. Numer. Meth. Engng., 73:869$894,2008$.

[10] F. Feyel and J.-L. Chaboche. Fe2 multiscale approach for modelling the elastoviscoplastic behavior of long fiber sic/ti composite materials. Comput. Meth. Appl. Mech. Engng., 183:309-330, 2000. 
[11] H. Moulinec and P. Suquet. A fast numerical method for computing the linear and nonlinear properties of composites. C. R. Acad. Sc. Paris II, 318:1417-1423, 1994.

[12] J. Yvonnet and Q. C. He. The reduced model multiscale method (R3M) for the non-linear homogenization of hyperelastic media at finite strains. J. Comput. Physics, 223:341-368, 2007.

[13] J. Aboudi. A continuum theory for fiber-reinforced elastic-viscoplastic composites. J. Eng. Sci., 20(55):605-621, 1982.

[14] M. Gosz, B. Moran, and J. D. Achenbach. Matrix cracking in transversely loaded fiber composites with compliant interphases. In AMD-150/AD-32, editor, Damage Mechanics in Composites. ASME, 1992.

[15] L. V. Berlyand and A. G. Kolpakov. Network approximation in the limit of small interparticle distance of the effective properties of a high-contrast random dispersed composite. Arch. Rational Mech. Anal., 159:179-227, 2001.

[16] G. J. Dvorak. Transformation field analysis of inelastic composite materials. Proc. R. Soc. Lond. A, 437:311-327, 1992.

[17] Y. A. Bahei-El-Din, A. M. Rajendran, and M. A. Zikry. A micromechanical model for damage progression in woven composite systems. Int. J. Solids Structures, 41:2307-2330, 2004 .

[18] J. Fish, Q. Yu, and K. L. Shek. Computational damage mechanics for composite materials based on mathematical homogenization. Int. J. Numer. Meth. Engng., 45:1657-1679, 1999.

[19] J. L. Chaboche, S. Kruch, J. F. Maire, and T. Pottier. Towards a micromechanics based inelastic and damage modeling of composites. Int. J. Plasticity, 17:411-439, 2001.

[20] J. C. Michel and P. Suquet. Computational analysis of nonlinear composite structures using the nonuniform transformation field analysis. Comput. Meth. Appl. Mech. Engng, 193:5477-5502, 2004.

[21] C. Oskay and J. Fish. Eigendeformation-based reduced order homogenization for failure analysis of heterogeneous materials. Comp. Meth. Appl. Mech. Engng., 196(7):1216-1243, 2007.

[22] K. Terada and N. Kikuchi. Nonlinear homogenization method for practical applications. In S. Ghosh and M. Ostoja-Starzewski, editors, Computational Methods in Micromechanics, volume AMD-212/MD-62, pages 1-16. ASME, 1995.

[23] J. T. Oden and S. Prudhomme. Goal-oriented error estimation and adaptivity for the finite element method. Computers \&5 Mathematics With Applications, 41:735-756, 2001.

[24] S. Ghosh, K. Lee, and P. Raghavan. A multi-level computational model for multi-scale damage analysis in composite and porous materials. Int. J. Solids Structures, 38:2335$2385,2001$.

[25] J. Nocedal and S. Wright. Numerical Optimization. Springer Verlag, 2nd edition, 2006.

[26] C. Oskay and J. Fish. On calibration and validation of eigendeformation-based multiscale models for failure analysis of heterogeneous systems. Comp. Mech., 42:181-195, 2008. 